\title{
A CONVERGENT SERIES EXPANSION FOR HYPERBOLIC SYSTEMS OF CONSERVATION LAWS
}

BY

\author{
EDUARD HARABETIAN ${ }^{1}$
}

\begin{abstract}
We consider the discontinuous piecewise analytic initial value problem for a wide class of conservation laws that includes the full three-dimensional Euler equations. The initial interaction at an arbitrary curved surface is resolved in time by a convergent series. Among other features the solution exhibits shock, contact, and expansion waves as well as sound waves propagating on characteristic surfaces. The expansion waves correspond to the one-dimensional rarefactions but have a more complicated structure. The sound waves are generated in place of zero strength shocks, and they are caused by mismatches in derivatives.
\end{abstract}

1. Introduction. We consider the initial value problem for a system of conservation laws given by

$$
\begin{aligned}
& u_{t}+\sum_{i=0}^{d}\left(f_{i}(u)\right)_{x_{i}}=0, \quad u, f_{i} \in \mathbf{R}^{n}, \\
& u\left(0, x_{0}, \ldots, x_{d}\right)= \begin{cases}u_{+}\left(x_{0}, \ldots, x_{d}\right), & x_{0}>S\left(x_{1}, \ldots, x_{d}\right), \\
u_{-}\left(x_{0}, \ldots, x_{d}\right), & x_{0}<S\left(x_{1}, \ldots, x_{d}\right),\end{cases}
\end{aligned}
$$

and satisfying the following:

(1) $f_{i}, S$ are analytic, $u_{+}, u_{-}$are analytic across $S$; however, $u\left(0, x_{0}, \ldots, x_{d}\right)$ may have a small jump discontinuity or a jump in derivatives, not necessarily small, at $S$.

(2) Equation (1.1) is hyperbolic in the following sense: If we let

$$
M(\omega, u)=\sum_{i=0}^{d} \omega_{i} \frac{\partial f_{i}}{\partial u}, \quad \omega \in \mathbf{R}^{d+1}-\{0\},
$$

then $M$ has real eigenvalues $\lambda_{1}(\omega, u) \leqslant \lambda_{2}(\omega, u) \leqslant, \ldots, \leqslant \lambda_{n}(\omega, u)$ and a basis of eigenvectors $r_{1}(\omega, u), \ldots, r_{n}(\omega, u)$. We denote left eigenvectors by $l_{i}(\omega, u)$.

(3) Equation (1.1) has either genuinely nonlinear or linearly degenerate fields, i.e.,

$$
\text { either } \nabla_{u} \lambda_{i} \cdot r_{i} \neq 0 \text { or } \nabla_{u} \lambda_{i} \cdot r_{i} \equiv 0
$$

for $u$ in a neighborhood of $u\left(0, x_{0}, \ldots, x_{d}\right)$ and $|\omega|=1$.

(4) If $M$ has multiple eigenvalues, then the corresponding field must be linearly degenerate.

Received by the editors March 11, 1985. Presented at the A.M.S. Annual Meeting Anaheim, California, January 1985 . Sponsored by I.C.A.S.E. NASA Langley Research Center

1980 Mathematics Subject Classification. Primary 35L65, 76N15, Secondary 35A10, 35A20.

Key words and phrases. Conservation laws, hyperbolic, Euler equations, majorization.

${ }^{1}$ Supported in part by NASA Contract No. NASI-17070. 
Our object is to obtain a power series representing a distribution solution to (1.1).

The conditions (2)-(4) are in part dictated by the properties characterizing the Euler equations. For a polytropic three-dimensional gas flow they are given by

$$
\left[\begin{array}{c}
\rho \\
\rho u \\
\rho v \\
\rho w \\
e
\end{array}\right]_{t}+\left[\begin{array}{c}
\rho u \\
\rho u^{2}+p \\
\rho u v \\
\rho u w \\
u(e+p)
\end{array}\right]_{x}+\left[\begin{array}{c}
\rho v \\
\rho u v \\
\rho v^{2}+p \\
\rho v w \\
v(e+p)
\end{array}\right]_{y}+\left[\begin{array}{c}
\rho w \\
\rho u w \\
\rho v w \\
\rho w^{2}+p \\
w(e+p)
\end{array}\right]_{z}=\left[\begin{array}{l}
0 \\
0 \\
0 \\
0 \\
0
\end{array}\right]
$$

with $p=(\gamma-1)\left(e-\left(\rho\left(u^{2}+v^{2}+w^{2}\right) / 2\right)\right)$ where $\rho=$ density, $u, v, w=$ velocities, $e=$ total energy, and $p=$ pressure [2].

The eigenvalues of $M$, in this case, are

$$
\omega_{0} u+\omega_{1} v+\omega_{2} w-c<\omega_{0} u+\omega_{1} v+\omega_{2} w<\omega_{0} u+\omega_{1} v+\omega_{2} w+c
$$

where $c=\sqrt{d p / d \rho}$ is the speed of sound in the medium. The first and third fields are genuinely nonlinear and the corresponding eigenvalues simple. The second field is linearly degenerate with the eigenvalue of multiplicity 3 . There is, however, a basis of eigenvectors so (2)-(4) are satisfied.

As a preliminary step we change variables to make the initial surface of discontinuity flat. If

$$
\begin{aligned}
x & =x_{0}-S\left(x_{1}, \ldots, x_{d}\right), \\
y_{i} & =x_{i}, \quad i=1,2, \ldots, d, \\
t & =t,
\end{aligned}
$$

then from (1.1)

$$
\begin{aligned}
& u_{t}+\left(f_{0}(u, y)\right)_{x}+\sum_{i=1}^{d}\left(f_{i}(u)\right)_{y_{i}}=0, \\
& u(0, x, y)= \begin{cases}u_{+}(x, y), & x>0, \\
u_{-}(x, y), & x<0,\end{cases}
\end{aligned}
$$

where by definition $f_{0}(u, y)=\sum_{i=0}^{d} f_{i}(u) \nu_{i}(y)$, with $\nu(y)$ normal to $S$.

The variables $t$ and $x$ will play the major role in our expansion with $y_{i}$ as parameters varying in the compact set $|y| \leqslant R_{0}$ for some $R_{0}$. The first term in the expansion will be given by the solution to the Riemann problem

$$
\begin{aligned}
& u_{t}+\left(f_{0}(u, y)\right)_{x}=0, \\
& u(0, x, y)= \begin{cases}u_{+}(0, y), & x>0, \\
u_{-}(0, y), & x<0 .\end{cases}
\end{aligned}
$$

If the system is strictly hyperbolic and the initial jump is small, the solution to the Riemann problem, due to P. D. Lax, is given in [1]. His proof involves the construction of the map $U\left(y, \varepsilon_{1}, \ldots, \varepsilon_{n}\right): \mathbf{R}^{n} \rightarrow \mathbf{R}^{n}$, with $y$ as a parameter, $U(y, 0, \ldots, 0)=u_{-}(0, y) . U\left(y, \varepsilon_{1}, \ldots, \varepsilon_{n}\right)$ represents the state obtained by starting from $u_{-}(0, y)$ and travelling $\varepsilon_{i}$ time increments along the appropriate shock, 
rarefaction, or contact curves. Lax obtains the solution by showing that $U$ is invertible near $\varepsilon=0$. The solution $u$ can be expressed as $u(t, x, y)=h(x / t, y)$ with $h\left(\lambda_{1}\left(u_{-}\right), y\right)=u_{-}$and $h\left(\lambda_{n}\left(u_{+}\right), y\right)=u_{+}$. The result immediately extends to the case with multiple eigenvalues in linearly degenerate fields if there is a basis of eigenvectors.

Our result in this paper is

TheOrem 1. Given $u_{-}(x, y)$, there exists $\varepsilon_{*}>0$ small and $C>0$ large, depending only on $u_{-}, f_{i}$, such that if $u_{+}(0, y)=U\left(y, \varepsilon_{1}, \ldots, \varepsilon_{n}\right), U(y, 0)=u_{-}(0, y)$ satisfies

(a) $\left|\varepsilon_{i}(y)\right| \leqslant \varepsilon_{*}, i=1,2, \ldots, n$,

(b) if $p$ is a genuinely nonlinear field then either (1) $\varepsilon_{p}(y) \neq 0$, for $|y| \leqslant R_{0}$, or $\left(1^{\prime}\right)$ $\varepsilon_{p}(y) \equiv 0$, for $|y| \leqslant R_{0}$, and

$$
\left|l_{p}\left(u_{-}(0, y)\right) \cdot\left(u_{-}\right)_{x}(0, y)-l_{p}\left(u_{+}(0, y)\right) \cdot\left(u_{+}\right)_{x}(0, y)\right| \geqslant C \varepsilon_{*},
$$

then we can construct a convergent power series which is a distribution solution to (1.2).

The solution consists of regions of analyticity separated by shock, contact, and rarefaction waves corresponding to the ones in the Riemann problem as well as sound waves corresponding to shocks of zero strength in the Riemann problem (the case $\varepsilon_{p} \equiv 0$ ). It therefore gives a precise description of the singularities propagating from the initial discontinuity (see Figure 1.1).

Condition (b) prevents shocks or rarefactions in the Riemann problem from degenerating to waves of zero strength within the parameter domain $|y| \leqslant R_{0}$, unless they are identically of zero strength. The difficulty with transitions to sound waves is caused by the fact that the two flat characteristic surfaces joining together in the Riemann problem will not necessarily ensure that the two curved characteristic surfaces in the full problem will likewise overlap one another.

One can distinguish between two types of regions, the ones in the "gaps" between waves where the solution is analytic in $x$ and $t$ and the ones in the rarefactions where it is analytic in the variable $x / t$. However, unlike the rarefactions in the Riemann problem, this last region is not a simple wave, in that characteristics are not flat and the solution is not constant along them.

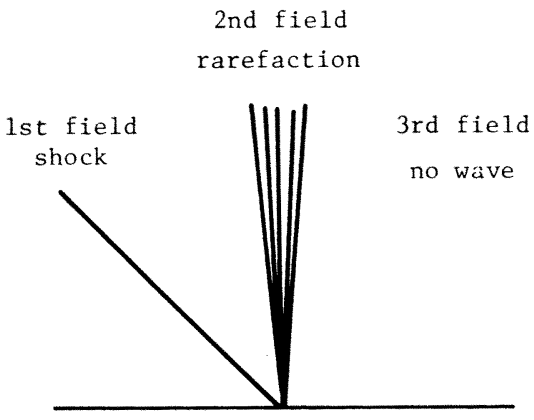

Riemann problem

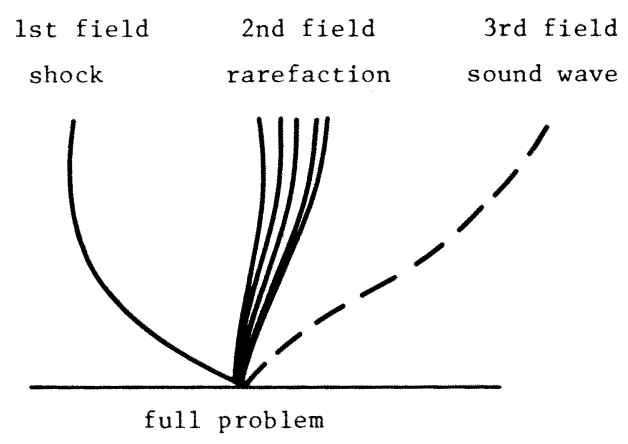

Figure 1.1 
The regions are separated by unknown surfaces where we impose the following boundary conditions: At rarefaction and sound surfaces we impose continuity across and given the existence of the coefficients of the expansion derive that the surfaces are characteristic as formal power series. Here we need condition (b)( $\left.1^{\prime}\right)$ to be able to determine the sound surface coefficients uniquely. At shock surfaces we impose the Rankine-Hugoniot conditions. At contacts we impose continuity of Riemann invariants and that the contact surface is characteristic. If the contact has a multiple eigenvalue, there will be less than $n$ equations imposed. Nevertheless, it can be shown that they imply all the Rankine-Hugoniot conditions across the contact.

The problem (1.1) with piecewise $H^{s}$ initial data restricted to ensure the formation of only one shock has been previously studied by A. Majda in [5] where the first stability and existence result for such systems with discontinuous initial data is given.

Theorem 1 answers a conjecture of R. D. Richtmyer on existence of solutions to hyperbolic systems of conservation laws with piecewise analytic initial data [6].

The proof consists of two parts. First, the coefficients are determined and estimated and, last, the expansion is shown to converge. In the first part we make appropriate changes of variables (\$2) which in the end only amount to rearrangements of power series. One could, just as easily, determine the coefficients of the original variables uniquely, but he would face enormous difficulties at the convergence step. To obtain the coefficients, we must solve algebraic equations in the gaps, $(n-1)$ linear ordinary differential equations coupled with one algebraic equation in rarefactions and coupling boundary equations at the shock, contact, rarefaction, and sound surfaces. This is accomplished in $\$ 3$. To show convergence we use the estimates in $\S 3$ to carry out the majorization argument in $\S 4$.

2. Expansions. Differentiating in (1.2) we obtain

$$
u_{t}+A(y, u) u_{x}+B(u) \cdot u_{y}=0
$$

with $A=\partial f_{0} / \partial u, B=\left(\partial f_{1} / \partial u, \ldots, \partial f_{d} / \partial u\right), u_{y}=\left(u_{y_{1}}, \ldots, u_{y_{d}}\right)$.

Let $A$ have $m$ distinct eigenvalues $\lambda_{1}<\lambda_{2}<\cdots<\lambda_{m}$ and let $\lambda_{p_{i}}, i=1, \ldots, s$, have multiplicity $\mu_{i}$ and correspond to the linearly degenerate fields. We choose a basis of eigenvectors so that $\nabla \lambda_{p} \cdot r_{p}=1$ in the genuinely nonlinear fields and $\left|r_{p_{i}}\right|=1$ in the linearly degenerate fields. If $\mu_{i}>1$ then there is a choice to be made in picking a basis for that eigenspace. We will adopt the following convention: In a linearly degenerate field, $r_{p_{i}}$ will refer to any of the eigenvectors $r_{p_{i}, 1}, \ldots, r_{p_{i}, \mu_{i}}$ that span the eigenspace. Similarly, in the expansion $u=\sum_{j=1}^{m} \alpha_{j} r_{j}, \quad \alpha_{p_{i}} r_{p_{i}}=$ $\sum_{k=1}^{\mu_{i}} \alpha_{p_{i}, k} r_{p_{i}, k}$, and $\alpha_{p_{i}}$ will refer to any of the components $\alpha_{p_{i}, 1}, \ldots, \alpha_{p_{i}, \mu_{i}}$.

Consider a gap (Figure 2.1) bounded on the left and right by

$$
\phi(t, y)=\lambda_{\phi}(y) t+\sum_{m=2}^{\infty} \phi_{m}(y) t^{m} \quad \text { and } \quad \psi(t, y)=\lambda_{\psi}(y) t+\sum_{n=2}^{\infty} \psi_{n}(y) t^{n}
$$

respectively. We change variables as follows:

$$
x=\phi(\xi, y)+\psi(\eta, y), \quad t=\xi+\eta, \quad y=y,
$$

where $\xi . \eta, y$ are the new gap variables. 


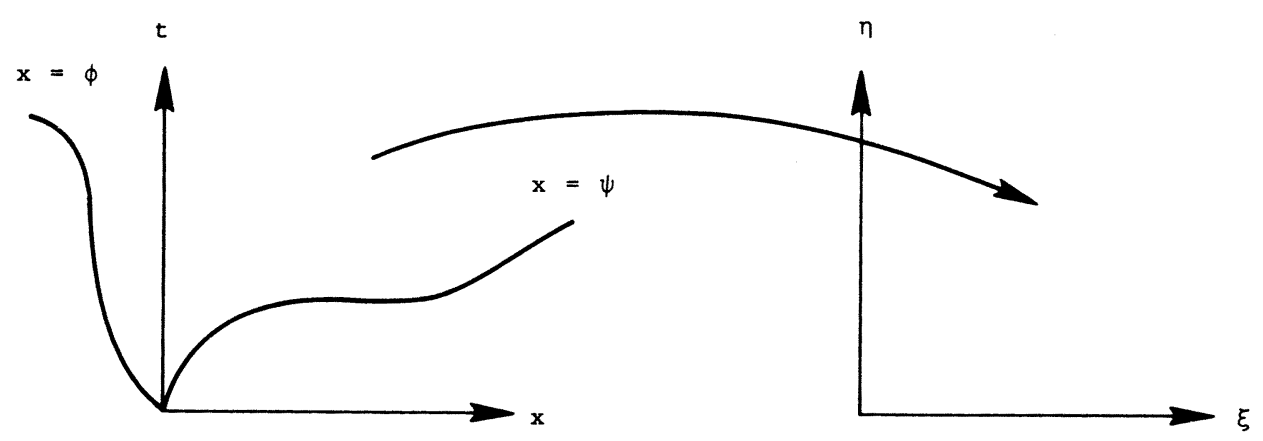

FIGURE 2.1

As shown in Figure $2.1 x=\phi, x=\psi$ are mapped into $\eta=0, \xi=0$, respectively.

We obtain that

$$
\frac{\partial(x, t, y)}{\partial(\xi, \eta, y)}=\left(\begin{array}{ccc}
\phi_{\xi} & \psi_{\eta} & \phi_{y}+\psi_{y} \\
1 & 1 & 0 \\
0 & 0 & I
\end{array}\right)
$$

with $\partial(x, t, y) / \partial(\xi, \eta, y)$ the Jacobian derivative, and therefore

$$
\operatorname{det}\left(\frac{\partial(x, t, y)}{\partial(\xi, \eta, y)}\left(0,0, y_{0}\right)\right)=\lambda_{\phi}-\lambda_{\psi} \neq 0
$$

Letting $u_{\text {new }}(\xi, \eta, y)=u_{\text {old }}(x, t, y)$, from (2.1)

$$
\begin{aligned}
\left(\psi_{\eta} I-A+(\psi+\phi)_{y} \cdot B\right) u_{\xi} \\
+\left(-\phi_{\xi} I+A-(\psi+\phi)_{y} \cdot B\right) u_{\eta}+\left(\psi_{\eta}-\phi_{\xi}\right) B \cdot u_{y}=0
\end{aligned}
$$

The end gaps, the first and the $(m+1)$ th (Figure 2.2$)$ are bounded by only one unknown surface.

Let $\lambda_{*}$ be a fixed number depending on $u_{-}$and $f_{i}, i=0, \ldots, d$. We will later specify how large $\lambda_{*}$ is.
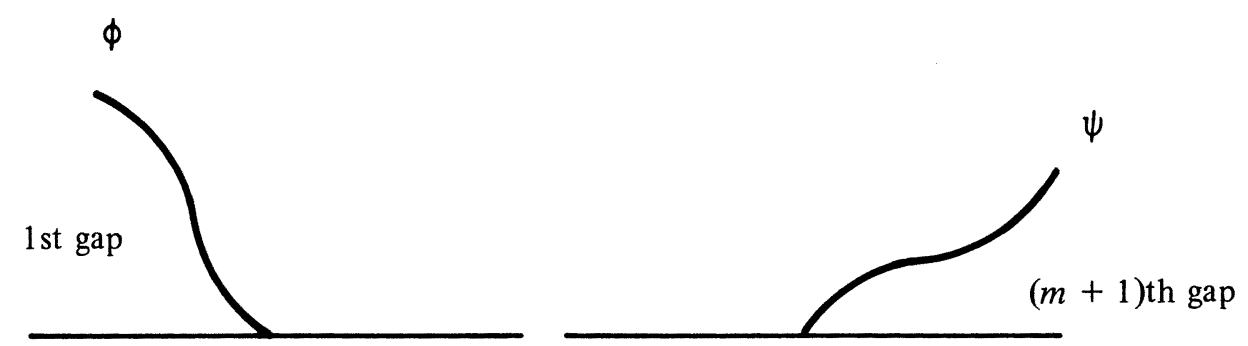

FIGURE 2.2 
In the first gap we let

Similarly in the $(m+1)$ th gap

$$
x=\phi(\eta, y)+\lambda_{*} \xi, \quad t=\eta, \quad y=y .
$$

$$
x=\psi(\xi, y)+\lambda_{*} \eta, \quad t=\xi, \quad y=y .
$$

We obtain from $(2.1)$

$$
u_{\eta}+\left(1 / \lambda_{*}\right)\left(A-\phi_{\eta}-\phi_{y} \cdot B\right) u_{\xi}+B \cdot u_{y}=0
$$

and

$$
u_{\xi}+\left(1 / \lambda_{*}\right)\left(A-\psi_{\xi}-\psi_{y} \cdot B\right) u_{\eta}+B \cdot u_{y}=0 .
$$

For a rarefaction bounded on the left and right by $\phi$ and $\psi$, respectively (Figure 2.3), we change variables as follows:

$$
s=\frac{x-\phi(t, y)}{\phi-\psi}, \quad t=t, \quad y=y .
$$

REMARKs. The Riemann solution was an analytic function of $x / t$ in rarefactions. Expanding the formula for $s$ above we get

$$
s=\frac{x / t-\phi / t}{(\phi-\psi) / t}=\frac{1}{\lambda_{\phi}-\lambda_{\psi}} \frac{x}{t}-\frac{\lambda_{\phi}}{\lambda_{\phi}-\lambda_{\psi}}+O(t),
$$

so $s$ behaves very much like $x / t$.

The transformation above maps $x=\phi, x=\psi$ to $s=0, s=1$, respectively.

In the new variables,

$$
\begin{gathered}
(\psi-\phi) u_{t}+\left\{A-\left(\phi_{t}+s(\psi-\phi)_{t}\right)-\left(\phi_{y}+s(\psi-\phi)_{y}\right) \cdot B\right\} u_{s} \\
+(\psi-\phi) B \cdot u_{y}=0 .
\end{gathered}
$$

REMARKS. As before $u_{\text {new }}(s, t, y)=u_{\text {old }}(x, t, y)$. Also, in (2.5), $A=A(u, y)$.

The solutions to (2.2), (2.5) are linked by boundary conditions. There are four types of boundaries: rarefaction, shock, sound, and contact.

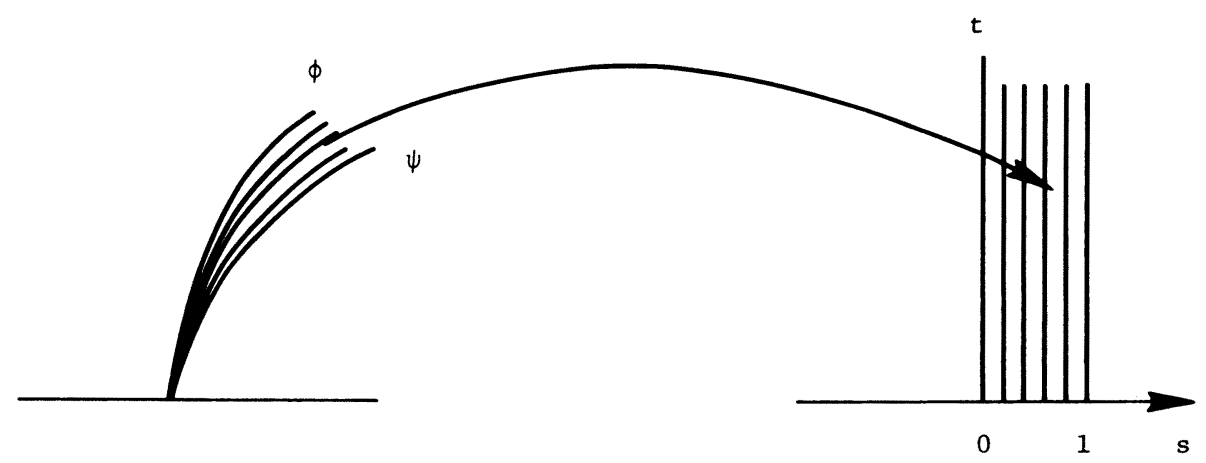

FigURE 2.3 


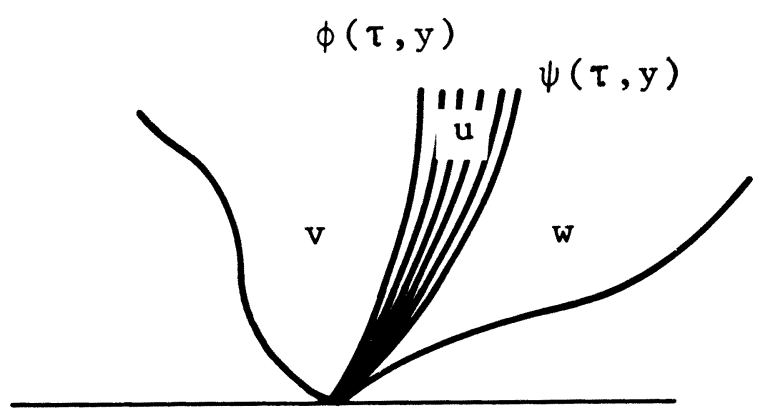

Figure 2.4

At a rarefaction surface we impose continuity, $u_{\text {old }}(\phi(\tau, y), \tau, y)=$ $v_{\text {old }}(\phi(\tau, y), \tau, y)$. In the new variables we get

$$
\begin{aligned}
& u(0, \tau, y)=v(0, \tau, y) \\
& u(1, \tau, y)=w(\tau, 0, y) \quad(\text { see Figure 2.4) }
\end{aligned}
$$

At a shock surface we impose the Rankine-Hugoniot conditions. For $v$ and $u$ on the left and right of a shock surface $\phi$, they are

$$
\phi_{\tau}(u-v)-\left(f_{0}(y, u)-f_{0}(y, v)\right)+\phi_{y}(f(u)-f(v))=0
$$

with $u=u(\tau, 0, y), v=v(0, \tau, y)$.

At a sound surface we impose continuity. For $u$ and $v$ as above and $\phi$ a sound surface, we get

$$
u(\tau, 0, y)=v(0, \tau, y)
$$

Consider now a contact surface $\phi(t, y)=\lambda_{p_{i}} t+\cdots$ in the linearly degenerate field $p_{i}$. To obtain weak solutions we should impose (2.7); however, if $\mu_{i}>1$, we expect that not all of the $n$ equations in (2.7) are independent.

For each $\tau, y$ we form the normal flux $-f_{0}+\phi_{y} f$ and the corresponding map $U\left(\varepsilon_{1}, \ldots, \varepsilon_{m}\right)$, analytic in $\varepsilon$ and built from rarefaction and contact curves only, such that $U(0, \ldots, 0)=u(\tau, 0, y)$. then $v(0, \tau, y)$ is connected to $u(\tau, 0, y)$ through a $p_{i}$ contact if and only if $v(0, \tau, y)=U\left(0, \ldots, \varepsilon_{p_{i}}, 0, \ldots, 0\right)$ for some $\varepsilon_{p_{i}}=$ $\left(\varepsilon_{p_{i}, 1}, \ldots, \varepsilon_{p_{i}, \mu_{i}}\right)$. A Riemann invariant for the $p_{i}$ th field is a function $R(u)$ such that $\nabla_{u} R \cdot r_{p_{i}} \equiv 0$ or equivalently $R\left(U\left(0, \ldots, 0, \varepsilon_{p_{i}}, 0, \ldots, 0\right)\right)=$ constant. There are exactly $n-\mu_{i}$ independent Riemann invariants. To obtain one such set we consider $Z_{1}(u), \ldots, Z_{n}(u)$ the coordinates of the inverse function of $U$ and let $R_{j}=Z_{j}$, $j \neq p_{i}$. Furthermore, we see that $\nabla R_{j} \cdot r_{k}=\delta_{j k}$ at $u=U(0,0, \ldots, 0)$ and $j, k \neq p_{i}$.

LEMMA 1. The $n-\mu_{i}+1$ conditions

$$
\begin{aligned}
& \text { (i) } \phi_{\tau}=\lambda_{p_{i}}\left(u, y, \phi_{y}\right), \\
& \text { (ii) } R_{j}\left(u, y, \phi_{y}\right)=R_{j}\left(v, y, \phi_{y}\right), \quad j \neq p_{i},
\end{aligned}
$$

imply that $\lambda_{p_{i}}\left(u, y, \phi_{y}\right)=\lambda_{p_{i}}\left(v, y, \phi_{y}\right)$ and that the Rankine-Hugoniot relations (2.7) hold. 
RemarKs. $\lambda_{p_{i}}(u, y, \omega)$ is the $p_{i}$ th eigenvalue of $-A(y, u)+\omega \cdot B(u) . R_{j}(u, y, \omega)$ is the $j$ th Riemann invariant for the flux $-f_{0}(y, u)+\omega \cdot f(u)$ and hence analytic in all of its arguments.

Proof. Fix $\tau, y$ and let $v=v(0, \tau, y)$. Then $v=U\left(\varepsilon_{1}, \ldots, \varepsilon_{m}\right)$ for some $\varepsilon_{1}, \ldots, \varepsilon_{m}$; consequently, (2.7b)(ii) $\Rightarrow \varepsilon_{j}=0$ for $j \neq p_{i}$. This means $v$ is connected to $u$ through a $p_{i}$ contact. Therefore $\lambda_{p_{i}}\left(u, y, \phi_{y}\right)=\lambda_{p_{i}}\left(v, y, \phi_{y}\right)$ and $\lambda_{p_{i}}(u-v)=\left(-f_{0}(y, u)+\right.$ $\left.\phi_{y} f(u)\right)-\left(-f_{0}(y, v)+\phi_{y} f(v)\right)$. The result follows from (2.7b)(i).

The Euler equations have two well-known Riemann invariants for the middle field. They are the pressure $p=(\gamma-1)\left(e-\left(\rho\left(u^{2}+v^{2}+w^{2}\right) / 2\right)\right)$ and the normal velocity $\hat{u}=\xi_{x} u+\xi_{y} v+\xi_{z} w$ with $\left(\xi_{x}, \xi_{y}, \xi_{z}\right)$ the normal to the surface. If, as before, the surface is given by $x=\phi(t, y, z)$ we have the following three conditions at the middle contact

$$
\begin{gathered}
\phi_{t}=-u_{0}+\phi_{y} v_{0}+\phi_{z} w_{0}, \\
-u_{0}+\phi_{y} v_{0}+\phi_{z} w_{0}=u_{1}+\phi_{y} v_{1}+\phi_{z} w_{1}, \\
p\left(\rho_{0}, u_{0}, v_{0}, w_{0}, e_{0}\right)=p\left(\rho_{1}, u_{1}, v_{1}, w_{1}, e_{1}\right),
\end{gathered}
$$

with $\left(\rho_{0}, u_{0}, v_{0}, w_{0}, e_{0}\right),\left(\rho_{1}, u_{1}, v_{1}, w_{1}, e_{1}\right)$ the left and right states. One easily verifies that (i) and (ii) above lead to the Rankine-Hugoniot conditions for the Euler equations. A tedious computation gives the eigenvectors

$$
r_{1}=\left[\begin{array}{c}
1 \\
u-\xi_{x} c \\
v-\xi_{y} c, \\
w-\xi_{z} c \\
H-\hat{u} c
\end{array}\right], \quad r_{3}=\left[\begin{array}{c}
1 \\
u+\xi_{x} c \\
v+\xi_{y} c \\
w+\xi_{z} c \\
H+u \hat{c}
\end{array}\right]
$$

corresponding to $\lambda_{1}=\hat{u}-c, \lambda_{3}=\hat{u}+c$, where the total enthalpy $H=(e+p) / \rho$ and the sound speed $c=\sqrt{d p / d \rho}$. It can now be easily verified that

$$
\left(\begin{array}{cc}
\nabla \hat{u} \cdot r_{1} & \nabla \hat{u} \cdot r_{3} \\
\nabla p \cdot r_{1} & \nabla p \cdot r_{3}
\end{array}\right)
$$

is invertible.

We seek power series solutions of the following form: In gaps

$$
u(\xi, \eta, y)=\sum_{m, n \geqslant 0} u_{m n}(y) \xi^{m} \eta^{n}
$$

whereas in rarefactions

$$
u(s, t, y)=\sum_{k \geqslant 0} u_{k}(s, y) t^{k}
$$

The first term in both series is obtained from the Riemann solution $h(s, y)$. In (2.8), $u_{00}(y)=h\left(\lambda_{\phi}, y\right)=h\left(\lambda_{\psi}, y\right)$ since $h$ is constant in its first variable in gaps. In (2.9), $u_{0}(s, y)$ is the solution to

$$
\left(A\left(u_{0}, y\right)-\left(\lambda_{\phi}+s\left(\lambda_{\psi}-\lambda_{\phi}\right)\right)\right)\left(u_{0}\right)_{s}=0
$$


which is the zero order relation obtained from substituting (2.9) into (2.5). It follows that

$$
u_{0}=h\left(s\left(\lambda_{\phi}-\lambda_{\psi}\right)+\lambda_{\phi}, y\right), \quad 0 \leqslant s \leqslant 1,
$$

and that if we have a $p$ rarefaction

$$
\lambda_{p}\left(u_{0}, y\right)=\lambda_{\phi}+s\left(\lambda_{\psi}-\lambda_{\phi}\right), \quad\left(u_{0}\right)_{s}=\left(\lambda_{\psi}-\lambda_{\phi}\right) r_{p}\left(u_{0}, y\right)
$$

The various unknown surfaces have expansions of the form

$$
\begin{aligned}
& \phi(\xi, y)=\lambda_{\phi}(y) \xi+\sum_{k \geqslant 2} \phi_{k}(y) \xi^{k} \\
& \psi(\eta, y)=\lambda_{\psi}(y) \eta+\sum_{k \geqslant 2} \psi_{k}(y) \eta^{k} .
\end{aligned}
$$

Substituting (2.8) and (2.11) into (2.2) and collecting terms for $\xi^{m} \eta^{n}$, we obtain the following recursive relations

$$
\left[\lambda_{\psi}-A\left(u_{00}\right)\right](m+1) u_{m+1, n}+\left[-\lambda_{\phi}+A\left(u_{00}\right)\right](n+1) u_{m, n+1}=F_{m n}
$$

with

$$
\begin{gathered}
F_{m n}=-\left[\left\{\left(\psi_{\eta}-\lambda_{\psi}\right) I-\left(A(u)-A\left(u_{00}\right)\right)+(\psi+\phi)_{y} B\right\} u_{\xi}\right. \\
+\left\{\left(\lambda_{\phi}-\phi_{\xi}\right)+\left(A(u)-A\left(u_{00}\right)\right)-(\psi+\phi)_{y} B\right\} u_{\eta} \\
\left.+\left(\psi_{\eta}-\phi_{\xi}\right) B \cdot u_{y}\right]_{m n} .
\end{gathered}
$$

REMARKS. $F_{m n}$ contains coefficients of $u$ of order $\leqslant m+n$ where by definition the order of $u_{m n}$ is $m+n$.

If we let $u_{m n}=\sum_{i=1}^{m}\left(\alpha_{i}\right)_{m n}(y) r_{i}\left(u_{00}, y\right)$, we obtain

$$
(m+1)\left[\lambda_{\psi}-\lambda_{i}\right]\left(\alpha_{i}\right)_{m+1, n}+(n+1)\left[-\lambda_{\phi}+\lambda_{i}\right]\left(\alpha_{i}\right)_{m, n+1}=\left(F_{i}\right)_{m n}
$$

where $\left(F_{i}\right)_{m n}=l_{i} \cdot(F)_{m n}, i=1,2, \ldots, m$, and $l_{i}\left(u_{00}, y\right), r_{i}\left(u_{00}, y\right), \lambda_{i}\left(u_{00}, y\right)$ are the left and right eigenvectors and eigenvalues of $A\left(u_{00}, y\right)$. For the end gaps (2.3) and (2.4) we obtain

$$
(n+1)\left(\alpha_{i}\right)_{m, n+1}+(m+1)\left(1 / \lambda_{*}\right)\left[\lambda_{i}-\lambda_{\phi}\right]\left(\alpha_{i}\right)_{m+1, n}=\left(F_{i}\right)_{m n}
$$

with

$$
\begin{gathered}
\left(F_{i}\right)_{m n}=l_{i}\left[\left(1 / \lambda_{*}\right)\left\{\left(\lambda_{\phi}-\phi_{\eta}\right)+\left(A(u)-A\left(u_{00}\right)\right)-\phi_{y} B\right\} u_{\xi}+B u_{y}\right]_{m n} \\
(m+1)\left(\alpha_{i}\right)_{m+1, n}+(n+1)\left(1 / \lambda_{*}\right)\left[\lambda_{i}-\lambda_{\psi}\right]\left(\alpha_{i}\right)_{m, n+1}=\left(F_{i}\right)_{m n}
\end{gathered}
$$

with

$$
\left(F_{i}\right)_{m n}=-l_{i}\left[\left(1 / \lambda_{*}\right)\left\{\left(\lambda_{\psi}-\psi_{\xi}\right)+\left(A(u)-A\left(u_{00}\right)\right)-\psi_{y} B\right\} u_{\eta}+B u_{y}\right]_{m n} .
$$

REMARKs. The reader will note the omission, for simplicity, of an index on the $L$ 's and $F$ 's signifying the gap we are in.

To obtain the equations in rarefactions we substitute (2.9) in (2.5) and collect the terms involving $t^{k}$ to get, for $k \geqslant 1$,

$$
\begin{gathered}
\left(\lambda_{\psi}-\lambda_{\phi}\right) k u_{k}+\left\{A\left(u_{0}\right)-\left(\lambda_{\phi}+s\left(\lambda_{\psi}-\lambda_{\phi}\right)\right)\right\}\left(u_{k}\right)_{s}+A^{\prime}\left(u_{0}\right) u_{k}\left(u_{0}\right)_{s} \\
-(k+1)\left(\phi_{k+1}+s\left(\psi_{k+1}-\phi_{k+1}\right)\right)\left(u_{0}\right)_{s}=F_{k}
\end{gathered}
$$


with

$$
\begin{aligned}
F_{k}=- & {\left[\left(\psi-\lambda_{\psi} t\right)-\left(\phi-\lambda_{\phi} t\right)\right\} u_{t}+\left(A(u)-A\left(u_{0}\right)\right)\left(u-u_{0}\right)_{s} } \\
+ & \left(A(u)-A\left(u_{0}\right)-A^{\prime}\left(u_{0}\right)\left(u-u_{0}\right)\right)\left(u_{0}\right)_{s} \\
& -\left(\phi_{t}-\lambda_{\phi}+s\left(\left(\psi_{t}-\lambda_{\psi}\right)-\left(\phi_{t}-\lambda_{\phi}\right)\right)\left(u-u_{0}\right)_{s}\right. \\
& \left.\left.+(\psi-\phi) B u_{y}-\left(\phi_{y}+s(\psi-\phi)_{y}\right)\right) B u_{s}\right]_{k} .
\end{aligned}
$$

REMARK. $\psi_{k}, \phi_{k}$ and $u_{k-1}$ are the highest order coefficients occurring in $F_{k}$.

If we substitute $u_{k}(s, y)=\sum_{i=1}^{m}\left(\alpha_{i}\right)_{k}(s, y) r_{i}\left(u_{0}, y\right)$ in (2.16) and use (2.10), we obtain

$$
\begin{aligned}
& \left(\lambda_{\psi}-\lambda_{\phi}\right) k\left(\alpha_{i}\right)_{k}+\left(\lambda_{i}\left(u_{0}\right)-\lambda_{p}\left(u_{0}\right)\right)\left(\alpha_{i_{s}}\right)_{k} \\
& \quad+\sum_{j=1}^{m}\left\{\left(\lambda_{i}-\lambda_{p}\right) l_{i} \cdot r_{j_{s}}+l_{i} \cdot\left(A^{\prime}\left(u_{0}\right) r_{j} u_{0_{s}}\right)\right\}\left(\alpha_{j}\right)_{k} \\
& \quad-(k+1)\left(\phi_{k+1}+s\left(\psi_{k+1}-\phi_{k+1}\right)\right)\left(\lambda_{\psi}-\lambda_{\phi}\right) \delta_{i p}=\left(F_{i}\right)_{k} .
\end{aligned}
$$

To simplify (2.18) we note that, by differentiating $A r_{p}=\lambda_{p} r_{p}$ with respect to $u_{r}$, multiplying on the left by $l_{i}$, on the right by $r_{j}$ and summing

$$
l_{i}\left(A^{\prime}\left(u_{0}\right) r_{j} u_{0_{s}}\right)=\left(\lambda_{\psi}-\lambda_{\phi}\right)\left(\lambda_{p}-\lambda_{i}\right) l_{i} J r_{p} r_{j}+\left(\lambda_{\psi}-\lambda_{\phi}\right) \delta_{i p} \nabla \lambda_{p} \cdot r_{j}
$$

with $J r_{p}=\partial r_{p} / \partial u$, the Jacobian derivative. Since

$$
l_{i} \cdot d r_{j} / d s=l_{i} \cdot J r_{j}\left(u_{0}\right)_{s}=\left(\lambda_{\psi}-\lambda_{\phi}\right) l_{i}\left(J r_{j}\right) r_{p}
$$

instead of (2.18), we now have

$$
\begin{array}{cc}
\left(\lambda_{i}-\lambda_{p}\right)\left(\alpha_{i_{s}}\right)_{k}+k\left(\lambda_{\psi}-\lambda_{\phi}\right)\left(\alpha_{i}\right)_{k}+\left(\lambda_{\psi}-\lambda_{\phi}\right) \sum_{j \neq p} \mathscr{B}_{j}\left(\alpha_{j}\right)_{k}= & \left(F_{i}\right)_{k} \\
(k+1)\left(\lambda_{\psi}-\lambda_{\phi}\right)\left(\alpha_{p}\right)_{k}+\left(\lambda_{\psi}-\lambda_{\phi}\right) \sum_{j \neq p}\left(\nabla \lambda_{p} \cdot r_{j}\right)\left(\alpha_{j}\right)_{k} & i \neq p \\
-(k+1)\left(\lambda_{\psi}-\lambda_{\phi}\right)\left(\phi_{k+1}+s\left(\psi_{k+1}-\phi_{k+1}\right)\right)=\left(F_{p}\right)_{k}
\end{array}
$$

where

$$
\mathscr{B}_{i j}=\left(\lambda_{i}-\lambda_{p}\right) l_{i} \cdot\left(\left(J r_{j}\right) r_{p}-\left(J r_{p}\right) r_{j}\right)
$$

Remarks. Note that $\mathscr{B}_{i p}=0$ which is why we let $j \neq p$ in the sum in (2.19). Since $\alpha_{p}$ does not appear in (2.19) we have a partial decoupling which will prove to be helpful.

We now turn to expansions at boundaries and use equations (2.6), (2.7), (2.7a), (2.7b). For rarefactions, from (2.6),

$$
u_{k}(0, y)=v_{0 k}(y), \quad u_{k}(1, y)=w_{k 0}(y) .
$$

For shocks we substitute series for $u, v, \phi$ into (2.7) and collect the coefficient of $\tau^{k}$ to obtain for $k \geqslant 1$

$$
\begin{aligned}
&(k+1) \phi_{k+1}\left(u_{00}-v_{00}\right)+\lambda_{\phi}\left(u_{k 0}-v_{0 k}\right) \\
&-\left(A\left(u_{00}\right) u_{k 0}-A\left(v_{00}\right) v_{0 k}\right)=g_{k}
\end{aligned}
$$


with

$$
\begin{aligned}
g_{k}=-\{( & \left.\phi_{\tau}-\lambda_{\phi}\right)\left(\left(u-u_{00}\right)-\left(v-v_{00}\right)\right) \\
- & {\left[\left(f_{0}(u)-f_{0}\left(u_{00}\right)-A\left(u_{00}\right)\left(u-u_{00}\right)\right)\right.} \\
& \left.\left.-\left(f_{0}(v)-f_{0}\left(v_{00}\right)-A\left(v_{00}\right)\left(v-v_{00}\right)\right)\right]+\phi_{y}(f(u)-f(v))\right\}_{k} .
\end{aligned}
$$

REMARKS. The zero order coefficient of $\tau$ is

$$
\lambda_{\phi}\left(u_{00}-v_{00}\right)-\left(f_{0}\left(u_{00}\right)-f_{0}\left(v_{00}\right)\right)=0
$$

which is the Rankine-Hugoniot condition for the zero order Riemann solution.

To simplify (2.21) we recall that for a $p$ shock $u_{00}=U\left(\varepsilon_{1}, \ldots, \varepsilon_{p}, 0, \ldots, 0\right)$ and $v_{00}=U\left(\varepsilon_{1}, \varepsilon_{2}, \ldots, \varepsilon_{p-1}, 0, \ldots, 0\right)$ with $\left(d / d \varepsilon_{p}\right) U\left(\varepsilon_{1}, \ldots, \varepsilon_{p-1}, 0,0, \ldots, 0\right)=r_{p}\left(v_{00}\right)$ [1]. As a result we have

(i) $l_{i}\left(v_{00}\right) \cdot r_{j}\left(u_{00}\right)=O\left(\varepsilon_{p}\right)$ if $i \neq j$;

(ii) $l_{i}\left(v_{00}\right) \cdot r_{i}\left(u_{00}\right)=1+O\left(\varepsilon_{p}\right)$;

(iii) $l_{p}\left(v_{00}\right) \cdot\left(u_{00}-v_{00}\right)=\varepsilon_{p}+O\left(\varepsilon_{p}^{2}\right)$;

(iv) $l_{i}\left(v_{00}\right) \cdot\left(u_{00}-v_{00}\right)=O\left(\varepsilon_{p}^{2}\right)$ if $i \neq p$.

By substituting

$$
u_{k 0}=\sum_{i}\left(\alpha_{j}\right)_{k 0} r_{j}\left(u_{00}\right), \quad v_{0 k}=\sum_{j}\left(\beta_{j}\right)_{0 k} r_{j}\left(v_{00}\right)
$$

in (2.21) and using (i)-(iv) above, we get

$$
\begin{aligned}
(k+1) \phi_{k+1} O\left(\varepsilon_{p}^{2}\right)+\left(\lambda_{\phi}-\lambda_{i}\left(u_{00}\right)\right)\left(\alpha_{i}\right)_{k 0}\left(1+O\left(\varepsilon_{p}\right)\right) \\
+O\left(\varepsilon_{p}\right) \sum_{j \neq i}\left(\lambda_{\phi}-\lambda_{j}\left(u_{00}\right)\right)\left(\alpha_{j}\right)_{k 0} \\
-\left(\lambda_{\phi}-\lambda_{i}\left(v_{00}\right)\right)\left(\beta_{i}\right)_{0 k}=\left(g_{i}\right)_{k}, \quad i \neq p,
\end{aligned}
$$

and

$$
\begin{aligned}
(k+1) \phi_{k+1}\left(\varepsilon_{p}\right. & \left.+O\left(\varepsilon_{p}^{2}\right)\right)+\left(\lambda_{\phi}-\lambda_{p}\left(u_{00}\right)\right)\left(\alpha_{p}\right)_{k 0}\left(1+O\left(\varepsilon_{p}\right)\right) \\
& +O\left(\varepsilon_{p}\right) \sum_{j \neq p}\left(\lambda_{\phi}-\lambda_{j}\left(u_{00}\right)\right)\left(\alpha_{j}\right)_{k 0} \\
& -\left(\lambda_{\phi}-\lambda_{p}\left(v_{00}\right)\right)\left(\beta_{p}\right)_{0 k}=\left(g_{p}\right)_{k}
\end{aligned}
$$

Since

$$
\begin{gathered}
\lambda_{p}\left(u_{00}\right)=\lambda_{p}\left(v_{00}\right)+O\left(\varepsilon_{p}\right), \quad \lambda_{\phi}-\lambda_{p}\left(u_{00}\right)=-\frac{\varepsilon_{p}}{2}+O\left(\varepsilon_{p}^{2}\right), \\
\lambda_{\phi}-\lambda_{p}\left(v_{00}\right)=\frac{\varepsilon_{p}}{2}+O\left(\varepsilon_{p}^{2}\right)
\end{gathered}
$$

we get

$$
\begin{aligned}
& (k+1) \phi_{k+1} O\left(\varepsilon_{p}^{2}\right)+\left(\lambda_{\phi}-\lambda_{i}\left(v_{00}\right)\right) \\
& \left.+\left(\alpha_{i}\right)_{k 0}-\left(\beta_{i}\right)_{0 k}\right) \\
& +O\left(\varepsilon_{p}\right) S_{i} \cdot(\alpha)_{k 0}=\left(g_{i}\right)_{k}, \quad i \neq p, \\
& (k+1) \phi_{k+1}\left(\varepsilon_{p}+O\left(\varepsilon_{p}^{2}\right)\right)+O\left(\varepsilon_{p}\right) S_{p} \cdot\left(\alpha_{p}\right)_{k 0}+O\left(\varepsilon_{p}\right) T \cdot\left(\beta_{p}\right)_{0 k}=\left(g_{p}\right)_{k}
\end{aligned}
$$


where $S_{j}=\left(S_{j 1}, S_{j 2}, \ldots, S_{j m}\right), j=1, \ldots, m, T=\left(T_{1}, \ldots, T_{m}\right)$ are vectors bounded independent of $\varepsilon_{p}$ near zero. $S$ and $T$ will change in the next equations, but they will remain bounded. Solve for $(k+1) \phi_{k+1}$ in the second equation to get

$$
(k+1) \phi_{k+1}=S_{p} \cdot(\alpha)_{k 0}+T \cdot(\beta)_{0 k}+\frac{1}{\varepsilon_{p}+O\left(\varepsilon_{p}^{2}\right)}\left(g_{p}\right)_{k} .
$$

Substitute in the first equation and divide by $\lambda_{\phi}-\lambda_{i}\left(v_{00}\right)$ to get

$$
\left(\alpha_{i}\right)_{k 0}-\left(\beta_{i}\right)_{0 k}+O\left(\varepsilon_{p}\right) S_{i} \cdot(\alpha)_{k 0}+O\left(\varepsilon_{p}\right) T \cdot(\beta)_{0 k}=P_{i} \cdot(g)_{k}, \quad i \neq p,
$$

where $P_{i}$ are bounded independent of $\varepsilon_{p}$ as well.

Let us now consider a sound surface $\phi=\lambda_{\phi} t+\cdots$, where $\lambda_{\phi}=\lambda_{p}\left(u_{00}\right)=$ $\lambda_{p}\left(v_{00}\right)$ and $\chi$ and $\psi$ are neighboring surfaces (see Figure 2.5). Expanding (2.7a) we simply get $u_{k 0}(y)=v_{0 k}(y)$ which in coordinates, since $u_{00}=v_{00}$, gives

$$
\left(\alpha_{i}\right)_{k 0}=\left(\beta_{i}\right)_{0 k} \text {. }
$$

The surface coefficients $\phi_{k}$ can be recovered from the gap relations (2.12). Focusing on the $p$ th equation, if we first let $m=n=0$ in (2.12), (2.13) we get

$$
\left(\lambda_{\psi}-\lambda_{p}\right)\left(\alpha_{p}\right)_{10}=-\left(\lambda_{\psi}-\lambda_{\phi}\right) l_{p} \cdot B\left(u_{00}\right)\left(u_{00}\right)_{y} .
$$

Similarly, in the left gap

$$
\left(\lambda_{p}-\lambda_{\chi}\right)\left(\beta_{p}\right)_{01}=-\left(\lambda_{\phi}-\lambda_{\chi}\right) l_{p} \cdot B\left(v_{00}\right)\left(v_{00}\right)_{y} .
$$

Since $u_{00}(y)=v_{00}(y)$ it follows that $\left(\alpha_{p}\right)_{10}=\left(\beta_{p}\right)_{01}$ is satisfied as a result of the gap equations. Next, letting $m=k-1>0, n=0$, note that from (2.12)

$$
F_{k-1,0}=k \phi_{k} u_{01}+k \phi_{k} B_{0} u_{00 y}+\tilde{F}_{k-1,0}
$$

where $\tilde{F}_{k-1,0}$ contains only lower order coefficients of $\phi$. Hence, from (2.13)

$$
k\left(\lambda_{\psi}-\lambda_{p}\right)\left(\alpha_{p}\right)_{k 0}=k \phi_{k} l_{p} \cdot\left(u_{01}+B_{0} u_{00 y}\right)+l_{p} \tilde{F}_{k-1,0} .
$$

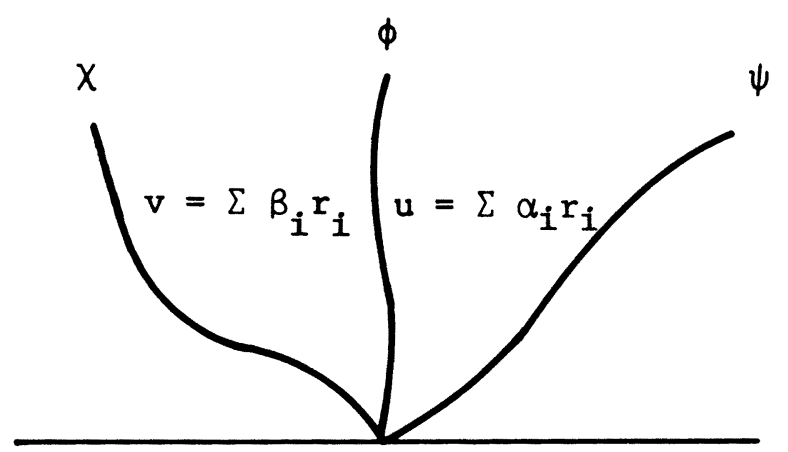

FIGURE 2.5 
Referring to the original variables, $x$ and $t$, for a moment

$$
\begin{aligned}
l_{p} \cdot u_{01} & =l_{p} \cdot\left[u_{t}(0,0)+\lambda_{\psi} u_{x}(0,0)\right] \\
& =l_{p} \cdot\left[-A\left(u_{00}\right) u_{x}(0,0)+\lambda_{\psi} u_{x}(0,0)-B\left(u_{00}\right) u_{00 y}\right] \\
& =\left(\lambda_{\psi}-\lambda_{p}\right) l_{p} \cdot u_{x}-l_{p} \cdot B_{0} u_{00 y}
\end{aligned}
$$

where we denote $u_{x}=u_{x}(0,0)$ in the gap. Hence,

$$
\left(\lambda_{\psi}-\lambda_{p}\right)\left(k\left(\alpha_{p}\right)_{k 0}-k \phi_{k} l_{p} \cdot u_{x}\right)=\tilde{F}_{k-1,0}
$$

Similarly, in the left gap

$$
\left(\lambda_{p}-\lambda_{\chi}\right)\left(k\left(\beta_{p}\right)_{0 k}-k \phi_{k} l_{p} \cdot v_{x}\right)=\tilde{F}_{0, k-1} .
$$

Therefore if $l_{p}\left(u_{x}-v_{x}\right) \neq 0$ the boundary condition $\left(\alpha_{p}\right)_{k 0}=\left(\beta_{p}\right)_{0 k}$ will determine $\phi_{k}$. To establish $l_{p}\left(u_{00}\right)\left(u_{x}-v_{x}\right) \neq 0$ we let $\phi$ be a shock surface, for example, and $w$ the function in the gap to the right of $u$. Expanding the shock relations

$$
\phi_{t}(w(\phi, t)-u(\phi, t))-\left[f_{0}\right]_{\text {jump at } \phi}+\phi_{y}[g]_{\text {jump at } \phi}=0
$$

and collecting first order terms we easily obtain

$$
l_{p}\left(u_{00}\right) u_{x}=l_{p}\left(w_{00}\right) w_{x}+O\left(\varepsilon_{*}\right) .
$$

Crossing a rarefaction will yield the same estimate by switching the sides and therefore reducing it to the shock case. Crossing a sound surface $u(\phi, t)=v(\phi, t)$, again gives the estimate above and, therefore, going through all the boundaries

$$
\begin{aligned}
& l_{p}\left(u_{00}\right) u_{x}=l_{p}\left(u_{+}(0,0)\right)\left(u_{+}\right)_{x}+O\left(\varepsilon_{*}\right), \\
& l_{p}\left(v_{00}\right) v_{x}=l_{p}\left(u_{-}(0,0)\right)\left(u_{-}\right)_{x}+O\left(\varepsilon_{*}\right) .
\end{aligned}
$$

If $C$ is large enough in (b)(1') of Theorem 1, we obtain the desired condition.

It remains to expand at contacts in $(2.7 \mathrm{~b})$. Equation $(2.7 \mathrm{~b})$ (ii) yields

$$
\nabla_{u} R_{j}\left(u_{00}, y, 0\right) \cdot u_{k 0}-\nabla_{v} R_{j}\left(v_{00}, y, 0\right) v_{0 k}=\left(L_{j}\right)_{k}
$$

where

$$
\begin{aligned}
\left(L_{j}\right)_{k}=- & \left\{\left(R_{j}\left(u, y, \phi_{y}\right)-R_{j}\left(u_{00}, y, 0\right)-\nabla R_{j}\left(u_{00}, y, 0\right)\left(u-u_{00}\right)\right)\right. \\
& \left.-\left(R_{j}\left(v, y, \phi_{y}\right)-R_{j}\left(v_{00}, y, 0\right)-\nabla R_{j}\left(v_{00}, y, 0\right)\left(v-v_{00}\right)\right)\right\}_{k} .
\end{aligned}
$$

Here we used the fact that $R_{j}\left(u_{00}, y, 0\right)=R_{j}\left(v_{00}, y, 0\right)$, i.e., the initial data are connected through a contact. If we let $u_{k 0}=\Sigma\left(\alpha_{i}\right)_{k 0} r_{i}\left(u_{00}\right), v_{0 k}=\Sigma\left(\beta_{i}\right)_{0 k} r_{i}\left(v_{00}\right)$ and use formulas (i)-(iv) derived for the shock expansions we get

$$
\sum_{i \neq p_{i}} \nabla R_{j}\left(u_{00}\right) \cdot r_{i}\left(u_{00}\right)\left(\left(\alpha_{i}\right)_{k 0}-\left(\beta_{i}\right)_{0 k}\right)+O\left(\varepsilon_{p_{i}}\right) S_{j}(\beta)_{0 k}=\left(L_{j}\right)_{k}
$$

with $S_{j}$ bounded independent of $\varepsilon_{p_{i}} \rightarrow 0$. Since $\left(\nabla R_{j}\left(u_{00}\right) \cdot r_{i}\left(u_{00}\right)\right), i, j \neq p_{i}$, is invertible,

$$
\left(\alpha_{i}\right)_{k 0}-\left(\beta_{i}\right)_{0 k}+O\left(\varepsilon_{p_{i}}\right) S_{i}(\beta)_{0 k}=P_{i} \cdot(L)_{k}
$$


with $S_{i}, P_{i}$ bounded matrices and $(L)_{k}=\left(\left(L_{j}\right)_{k}, j \neq p_{i}\right)$. From $(2.7 \mathrm{~b})(\mathrm{i})$

$$
\begin{aligned}
& (k+1) \phi_{k+1}=\sum_{j=1}^{m}\left(\nabla_{u} \lambda_{p_{i}}\left(u_{00}, y, 0\right) \cdot r_{j}\left(u_{00}\right)\right)\left(\alpha_{j}\right)_{k 0}+\left(L_{P_{i}}\right)_{k}, \\
& \left(L_{p_{i}}\right)_{k}=\left\{\lambda_{p_{i}}\left(u, y, \phi_{y}\right)-\lambda_{p_{i}}\left(u_{00}, y, 0\right)-\nabla_{u} \lambda_{p_{i}}\left(u_{00}, y, 0\right) \cdot\left(u-u_{00}\right)\right\}_{k}
\end{aligned}
$$

for $k \geqslant 1$.

At this point we should be able to show that all coefficients can be uniquely determined from the formulas established so far. We will do it in the next section. To conclude this section, we derive from the conditions already imposed that the rarefaction and sound surfaces satisfy a characteristic equation.

We have

LEMMA 2. Suppose there is a unique formal power series solution. If $\phi$ is either a p-rarefaction surface or a p-sound surface, then

$$
\left(\phi_{t}(t, y)\right)_{k}=\left(\lambda_{p}\left(u, y, \phi_{y}\right)\right)_{k}=\left(\lambda_{p}\left(v, y, \phi_{y}\right)\right)_{k}
$$

with $u, v$ the solutions near $\phi$.

Proof. We give the argument for rarefactions, the one for sound surfaces following the same lines.

Suppose $u$ is the function in the rarefaction to the right of $\phi$ and $v$ is in the gap on the left. Let

$$
u_{\text {old }}(x, t, y)=H(x / t, t, y), \quad v_{\text {old }}(x, t, y)=G(x / t, t, y) .
$$

Then $u_{\text {new }}(s, t, y)=H(s((\phi-\psi) / t)+\phi / t, t, y)$ and $H(\sigma, t, s), G(\sigma, t, y)$ satisfy

$$
\begin{gathered}
t H_{t}+(A-\sigma I) H_{\sigma}+t B H_{y}=0, \\
t G_{t}+(A-\sigma I) G_{\sigma}+t B G_{y}=0,
\end{gathered}
$$

and $H(\phi / t, t, y)=G(\phi / t, t, y)$. Differentiating and multiplying by $t$

$$
\begin{aligned}
t H_{t}+H_{\sigma}\left(\phi_{t}-\phi / t\right) & =t G_{t}+G_{\sigma}\left(\phi_{t}-\phi / t\right), \\
H_{y_{i}}+H_{\sigma} \phi_{y_{i}} / t & =G_{y_{i}}+G_{\sigma} \phi_{y_{i}} / t .
\end{aligned}
$$

Using (2.27a) and (2.27b) with $s=\phi / t$ the first equation leads to

$$
\begin{aligned}
& H_{\sigma}\left(\phi_{t}-\phi / t\right)-(A-\phi(t, y) / t) H_{\sigma}-t B H_{y} \\
& \quad=G_{\sigma}\left(\phi_{t}-\phi / t\right)-(A-\phi / t) G_{\sigma}-t B G_{y} .
\end{aligned}
$$

The second equation, after multiplying by $t B_{i}$ and adding, yields

$$
\phi_{y} B H_{\sigma}+t B H_{y}=\phi_{y} B G_{\sigma}+t B G_{y} \text {. }
$$

Hence we obtain

$$
\left(\phi_{t}-A+\phi_{y} B\right) H_{\sigma}=\left(\phi_{t}-A+\phi_{y} B\right) G_{\sigma} .
$$

Multiplying on the left by $l_{p}\left(H(\phi / t, t, y), y, \phi_{y}\right)=l_{p}\left(G(\phi / t, t, y), y, \phi_{y}\right)$, we get

$$
\left(\phi_{t}-\lambda_{p}\left(u, y, \phi_{y}\right)\right) l_{p} \cdot\left(H_{\sigma}-G_{\sigma}\right)=0 \text {. }
$$


Now $\left(l_{p} \cdot\left(H_{\sigma}-G_{\sigma}\right)\right)_{0}=l_{p}\left(h\left(\lambda_{\phi}, y\right), y\right) \cdot h_{\sigma}\left(\lambda_{\phi}, y\right)=1$, where $h(\sigma, y)=$ $H\left(\lambda_{\phi}, 0, y\right)$ is the Riemann solution, and since $G$ is in the gap $\left(G_{\sigma}\right)_{0}=G_{\sigma}\left(\lambda_{\phi}, 0, y\right)$ $=0$. Also $h_{\sigma}\left(\lambda_{\phi}, y\right)=r_{p}\left(h\left(\lambda_{\phi}, y\right), y\right)$. Therefore, since

$$
\left(\phi_{t}-\lambda_{p}\left(H, y, \phi_{y}\right)\right)_{k}+\sum_{\mu=0}^{k-1}\left(\phi_{t}-\lambda_{p}\right)_{\mu}\left(l_{p} \cdot\left(H_{\sigma}-G_{\sigma}\right)\right)_{k-\mu}=0
$$

and $\left(\phi_{t}-\lambda_{p}\right)_{0}=0$, our claim (2.26) follows by induction.

REMARK. Note that $H(\phi / t, t, y)=u(0, t, y)$.

Expanding (2.26) we get

$$
\begin{aligned}
\lambda_{\phi}+2 \phi_{2} t+3 \phi_{3} t^{2}+ & \cdots+(k+1) \phi_{k+1} t^{k}+\cdots \\
& =\lambda_{p}\left(u_{0}, y, 0\right)+\nabla_{u} \lambda_{p} \cdot\left(u-u_{0}\right)+L_{p}\left(u, y, \phi_{y}\right)
\end{aligned}
$$

where $L_{p}\left(u_{0}, y, 0\right)=0$ and $L_{p}$ is quadratic in $\left(u-u_{0}\right)$. Hence, for $k \geqslant 1$, we get

$$
(k+1) \phi_{k+1}(y)=\sum_{i=1}^{m}\left(\nabla_{u} \lambda_{p}(0, y) \cdot r_{i}\right)\left(\alpha_{i}\right)_{k}(0, y)+\left(L_{p}\right)_{k}
$$

REMARK. $\left(L_{p}\left(u, y, \phi_{y}\right)\right)_{k}$ contains $u_{k+1}, \phi_{k}$ as highest order coefficients. In fact

$$
\left(L_{p}\right)_{k}=\left(\lambda_{p}\left(u, y, \phi_{y}\right)-\lambda_{p}\left(u_{00}, y, 0\right)-\nabla_{u} \lambda_{p}\left(u_{00}, y, 0\right) \cdot\left(u-u_{00}\right)\right)_{k} .
$$

Formulas (2.28), (2.29) are, in fact, expansions valid for rarefactions, sound, as well as contact surfaces (see $(2.25 \mathrm{c})$ ).

3. Linear estimates. In this section we derive a priori estimates for the linear system of equations satisfied by the $k$ th order coefficients with inhomogeneous terms depending on lower order coefficients. These estimates will help in determining the coefficients uniquely and subsequently in showing the series converges.

In (2.13), to obtain coefficients of order $k$, we take $m+n+1=k(k \geqslant 1)$. As (2.20), (2.23), (2.25c) suggest, we would expect to determine $\phi_{k+1}$ for shocks, contacts, and rarefactions at the same time we determine $\alpha_{k}$ 's. For sound surfaces we can only determine $\phi_{k}$ from the boundary conditions, but (2.28) shows that, once determined, the coefficients $\phi_{k}$ can be estimated at the previous step.

Consider the diagram in Figure 3.1 showing the $m$ fields with the gaps between them. We let dotted lines signify the various waves. For example, in Figure 3.1 we collapse a $p$-rarefaction to a dotted line with arrows pointing at corresponding faces.
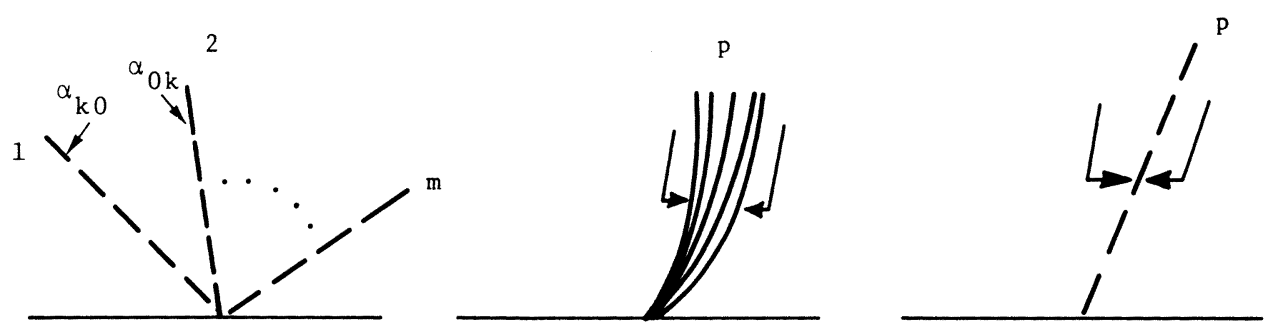

FIGURE 3.1 
We now want to consider the coefficients in the gaps at the boundaries of the gaps. From (2.8), $\alpha_{k 0}$ are the coefficients of the expansion at $\eta=0$, the left boundary of the gap. Similarly, $\alpha_{0 k}$ are the coefficients at the right boundary. In the first gap we only consider $\alpha_{0 k}$, at the right boundary and in the $(m+1)$ th gap $\alpha_{k 0}$, the left boundary.

Since there are $m$ boundaries, each with $2 n$ components on both sides, we get a total of $2 m n$ unknown boundary components. They satisfy a linear system of equations given by the gap equations (2.13)-(2.15) and by boundary equations: rarefactions (2.20), shocks (2.24), sound (2.24a), and contacts $(2.25 \mathrm{~b})$. Note that the $p$ th equation at each boundary determines the surface coefficient. For example, at a sound surface, $\phi_{k}$ is determined from the $p$ th equation at the boundary and, in view of $(2.24 b),(2.24 c)$, it can be solved in terms of lower order terms and hence substituted back into the equations (2.12) for the neighboring gaps. (For $k=1$ the $p$ th equation is satisfied automatically.) As a second example, the $p$ th equation at the boundary of a rarefaction region (the continuity condition) determines $\left(\alpha_{p}\right)_{k}(0, y),\left(\alpha_{p}\right)_{k}(1, y)$. They, in turn, determine $\phi_{k+1}$ and $\psi_{k+1}$ by evaluating (2.20) at $s=0$ and $s=1$. Fortunately, as we mentioned in the remark after (2.20), we can solve (2.19) independent of $\left(\alpha_{p}\right)_{k 0}$. We may therefore only consider the $n-\mu_{p}$ equations at the $p$ boundary. If $p$ is a genuinely nonlinear field, $\mu_{p}=1$ and we have $n-1$ equations. The total number of equations for the $2 m n$ unknowns is

$$
\left(\sum_{i=1}^{m} n-\mu_{i}\right)+(m+1) n=2 m n \text {. }
$$

The first term above gives the total number of equations from boundaries, the second one gives the total number from gaps. To show the system has a unique solution it suffices to prove the linear mapping is one to one. This will follow from the estimates ahead.

We now divide the unknowns into two groups $\vec{a}_{k}$ and $\vec{b}_{k}$. If we are at the $p$ th boundary (dotted line), we count $\left(\alpha_{1}, \ldots, \alpha_{p-1}\right)$ in the gap on the left and $\left(\alpha_{p+1}, \ldots, \alpha_{m}\right)$ in the gap on the right as part of $\vec{a}$ and $\vec{a}$ consists of exactly these components. The rest forms $\vec{b}$ (see Figure 3.2). It follows that $\vec{a}$ has $n \cdot(m-1)$ components and $\vec{b}$ has $n \cdot(m+1)$ components.

We will be able to estimate $\vec{a}$ from the boundary equations and $\vec{b}$ from the gap equations. In rarefactions $\alpha_{p}$ is the characteristic component satisfying the algebraic

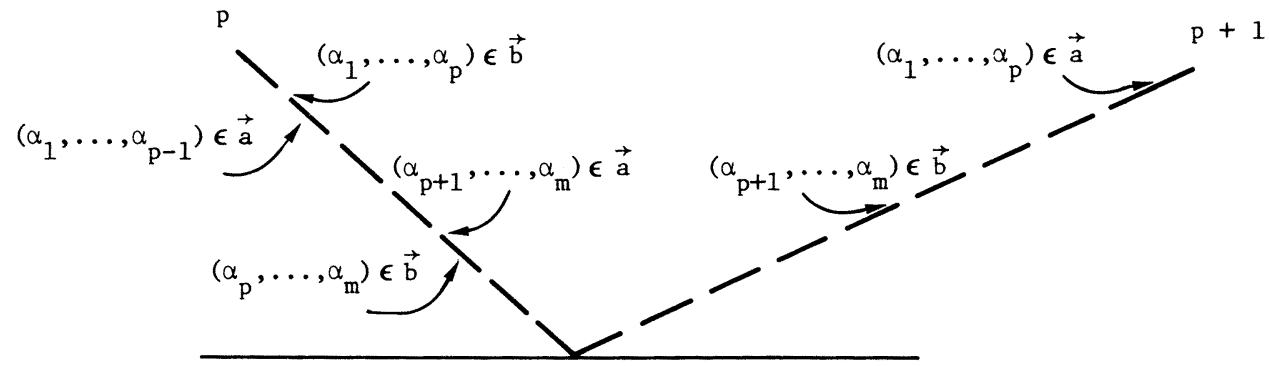

FIGURE 3.2 
relation in (2.20). Note that since the boundary values of $\alpha_{p}$ belong to $\vec{b}$ and not $\vec{a}$, they will be estimated from the gap equations and not from (2.20).

(A) Estimates from gaps. We consider the gap between the $p$ th and $(p+1)$ th fields bounded by surfaces $\phi$ and $\psi$ on the left and right, respectively. Lax's entropy conditions give $\lambda_{p+1}\left(u_{00}\right) \geqslant \lambda_{\psi}>\lambda_{\phi} \geqslant \lambda_{p}\left(u_{00}\right)$. We get equalities at the ends for sound or rarefaction surfaces. From (2.13) we get

$$
\begin{aligned}
\left(\alpha_{i}\right)_{m+1, n}= & \left(\frac{n+1}{m+1}\right)\left(\frac{\lambda_{\phi}-\lambda_{i}}{\lambda_{\psi}-\lambda_{i}}\right)\left(\alpha_{i}\right)_{m, n+1}+\frac{1}{m+1} \frac{1}{\lambda_{\psi}-\lambda_{i}}\left(F_{i}\right)_{m, n}, \quad i \leqslant p, \\
\left(\alpha_{i}\right)_{m, n+1}=\left(\frac{m+1}{n+1}\right)\left(\frac{\lambda_{i}-\lambda_{\psi}}{\lambda_{i}-\lambda_{\phi}}\right)\left(\alpha_{i}\right)_{m+1, n}+\frac{1}{n+1} \frac{1}{\lambda_{i}-\lambda_{\phi}}\left(F_{i}\right)_{m, n}, & i \geqslant p+1 .
\end{aligned}
$$

Let

$$
\rho_{i}= \begin{cases}\left(\lambda_{\phi}-\lambda_{i}\right) /\left(\lambda_{\psi}-\lambda_{i}\right), & i \leqslant p \\ \left(\lambda_{i}-\lambda_{\psi}\right) /\left(\lambda_{i}-\lambda_{\phi}\right), & i \geqslant p+1\end{cases}
$$

Then there exits $\rho$ independent of $\varepsilon_{*}$ near zero but depending on $u_{-}(y), f_{i}$ such that $0<\rho_{i}(y) \leqslant \rho<1$ for $\left|y_{i}\right| \leqslant R_{0}$ where $u_{+}(y)=U\left(\varepsilon_{1}, \varepsilon_{2}, \ldots, \varepsilon_{m}\right)$ and $\left|\varepsilon_{i}\right| \leqslant \varepsilon_{*}$, with $\varepsilon_{*}$ to be chosen. We claim we have

$$
\begin{aligned}
\left(\alpha_{i}\right)_{m+1, n}= & \rho_{i}^{m+1}\left(\begin{array}{c}
m+n+1 \\
n
\end{array}\right)\left(\alpha_{i}\right)_{0 k} \\
& +\rho_{i}^{m} \cdot \frac{(n+1)(n+2) \cdots(n+m)}{(m+1) \cdot m \cdots 2 \cdot 1} \frac{1}{\lambda_{\psi}-\lambda_{i}}\left(F_{i}\right)_{0, m+n} \\
& +\rho_{i}^{m-1} \cdot \frac{(n+1) \cdots(n+m-1)}{(m+1) \cdot m \cdots 2} \frac{1}{\lambda_{\psi}-\lambda_{i}}\left(F_{i}\right)_{1, m+n-1}+\cdots \\
& +\rho_{i} \frac{(n+1)}{(m+1) \cdot m} \frac{1}{\lambda_{\psi}-\lambda_{i}}\left(F_{i}\right)_{m-1, n+1}+\frac{1}{\lambda_{\psi}-\lambda_{i}} \frac{1}{m+1}\left(F_{i}\right)_{m, n}
\end{aligned}
$$

for $i \leqslant p$

and

$$
\begin{aligned}
\left(\alpha_{i}\right)_{m, n+1}= & \rho_{i}^{n+1}\left(\begin{array}{c}
m+n+1 \\
m
\end{array}\right)\left(\alpha_{i}\right)_{k 0} \\
& +\rho_{i}^{n} \frac{(m+1)(m+2) \cdots(m+n)}{(n+1) \cdots 2 \cdot 1} \frac{1}{\lambda_{i}-\lambda_{\phi}}\left(F_{i}\right)_{m+n, 0} \\
& +\cdots+\frac{1}{\lambda_{i}-\lambda_{\phi}} \frac{1}{(n+1)}\left(F_{i}\right)_{m, n} \quad \text { for } i \geqslant p+1
\end{aligned}
$$

This can be proved inductively on $m$, say, by substituting formulas (3.1a) for $\left(\alpha_{i}\right)_{m, n+1}$ into the recursion formula for $\left(\alpha_{i}\right)_{m+1, n}$. 
For notational convenience we let $\left(\mathscr{F}_{i}\right)_{m n}$ be the sums involving the $F_{i}$ 's on the right-hand side of (3.1a) and (3.1b). Hence (3.1a) and (3.1b) can be written as

$$
\begin{array}{ll}
\left(\alpha_{i}\right)_{m+1, n}=\rho_{i}^{m+1}\left(\begin{array}{c}
m+n+1 \\
n
\end{array}\right)\left(\alpha_{i}\right)_{0, k}+\left(\mathscr{F}_{i}\right)_{m, n}, & i \leqslant p, \\
\left(\alpha_{i}\right)_{m, n+1}=\rho_{i}^{n+1}\left(\begin{array}{c}
m+n+1 \\
m
\end{array}\right)\left(\alpha_{i}\right)_{k, 0}+\left(\mathscr{F}_{i}\right)_{m, n}, & i \geqslant p+1,
\end{array}
$$

which gives

$$
\begin{gathered}
\left(\alpha_{i}\right)_{k, 0}=\rho_{i}^{k}\left(\alpha_{i}\right)_{0, k}+\left(\mathscr{F}_{i}\right)_{k-1,0}, \quad i \leqslant p, \\
\left(\alpha_{i}\right)_{0, k}=\rho_{i}^{k}\left(\alpha_{i}\right)_{k, 0}+\left(\mathscr{F}_{i}\right)_{0, k-1}, \quad i \geqslant p+1 .
\end{gathered}
$$

In the end gaps we have

1st gap

$$
u_{\text {new }}(0, \xi, y)=u_{\text {old }}\left(\lambda_{*} \xi, 0, y\right)=u_{-}\left(\lambda_{*} \xi, 0, y\right),
$$

$(m+1)$ th gap

$$
u_{\text {new }}(\eta, 0, y)=u_{\text {old }}\left(\lambda_{*} \eta, 0, y\right)=u_{+}\left(\lambda_{*} \eta, 0, y\right) \text {. }
$$

Let $\left(u_{ \pm}\left(\lambda_{*} \xi, 0, y\right)\right)_{k}=\sum\left(\alpha_{ \pm i}\right)_{k} r_{i}\left(u_{ \pm 00}\right)$ with $u_{ \pm 00}=u \pm(0,0, y)$. Then from (2.14) and (2.15) we get the following relations for the end gaps, 1st gap, and $(m+1)$ th gap, respectively:

$$
\begin{aligned}
& \left(\alpha_{i}\right)_{m, n+1}=\rho_{i}^{n+1}\left(\begin{array}{c}
m+n+1 \\
m
\end{array}\right)\left(\alpha_{-i}\right)_{k}+\left(\mathscr{F}_{i}\right)_{m, n} \text { all } i, \\
& \left(\alpha_{i}\right)_{m+1, n}=\rho_{i}^{m+1}\left(\begin{array}{c}
m+n+1 \\
n
\end{array}\right)\left(\alpha_{+_{i}}\right)_{k}+\left(\mathscr{F}_{i}\right)_{m, n} \quad \text { all } i .
\end{aligned}
$$

Here $\rho_{i}=\left(\lambda_{\phi}-\lambda_{i}\right) / \lambda_{*}$ and it follows that if we pick $\lambda_{*}$ large enough, depending only on $u_{-}(y), f_{i}$, we have $0<\left|\rho_{i}\right| \leqslant \rho<1$. Hence it follows that

$$
\begin{gathered}
\left(\alpha_{i}\right)_{0, k}=\rho_{i}^{k}\left(\alpha_{-i}\right)_{k}+\left(\mathscr{F}_{i}\right)_{0, k-1}, \quad \text { 1st gap, } \\
\left(\alpha_{i}\right)_{k, 0}=\rho_{i}^{k}\left(\alpha_{+_{i}}\right)_{k}+\left(\mathscr{F}_{i}\right)_{k-1,0}, \quad(m+1) \text { st gap. }
\end{gathered}
$$

Let $\mathscr{F}_{k-1}$ denote the vector containing all $\mathscr{F}_{i}$ 's from all gaps (3.2), (3.3), (3.6), (3.7). We note that the components on the left-hand side of (3.2), (3.3), (3.6), (3.7) together form the whole of $\vec{b}_{k}$ and the ones on the right-hand side of (3.2), (3.3) next to the $\rho_{i}^{k}$ form the whole of $\vec{a}_{k}$.

Hence (3.2), (3.3), (3.6), (3.7) give us

$$
\left|\vec{b}_{k}\right| \leqslant \rho^{k}\left|\vec{a}_{k}\right|+\left|\left(\alpha_{+}\right)_{k}\right|+\left|\left(\alpha_{-}\right)_{k}\right|+\left|\mathscr{F}_{k-1}\right|
$$

where $\left|\vec{b}_{k}\right|=\max _{i}\left\{\left|b_{i k}\right|\right\}$ denotes the max norm.

REMARKS. $\left|\mathscr{F}_{k-1}\right|=\max _{i} \mid F_{i}$ 's $\mid$ and by $\mathscr{F}_{i}$ 's we understand $\left(\mathscr{F}_{i}\right)_{k 0}$ or $\left(\mathscr{F}_{i}\right)_{0 k}$ as the case may be.

(B) Estimates from rarefactions. We consider (2.19) with $0 \leqslant s \leqslant 1$. Let

$$
\begin{gathered}
\Lambda_{i}(\sigma, y)=\left(\lambda_{\psi}(y)-\lambda_{\phi}(y)\right) /\left(\lambda_{p}(\sigma, y)-\lambda_{i}(\sigma, y)\right), \quad i \leqslant p-1, \\
\Lambda_{i}(\sigma, y)=\left(\lambda_{\psi}-\lambda_{\phi}\right) /\left(\lambda_{i}-\lambda_{p}\right), \quad i \geqslant p+1 .
\end{gathered}
$$

Then if $\varepsilon_{p}=\varepsilon_{p}(y)$ is such that $u_{+}(y)=U\left(y, \varepsilon_{1}, \ldots, \varepsilon_{p}, \ldots, \varepsilon_{m}\right)$ we have $\varepsilon_{p}(y)=$ $\lambda_{\psi}(y)-\lambda_{\phi}(y)$ and hence

$$
0<\varepsilon_{0} \leqslant \Lambda_{i} \leqslant C_{0} \varepsilon_{*}, \quad\left|y_{i}\right| \leqslant R,
$$


with $\varepsilon_{0}=\inf \left\{\Lambda_{i},\left|y_{i}\right| \leqslant R_{0}\right\}>0$ by (b)(1) of Theorem 1, and $C_{0}$ dependent on $u_{-}$, $f_{i}$ only. For $i \leqslant p-1$ we use $\exp \left(k \int_{s}^{1} \Lambda_{i}(\sigma, y) d \sigma\right)$ as an integrating factor. For $i \geqslant p+1$ we use $\exp \left(k \int_{0}^{s} \Lambda_{i}(\sigma, y) d \sigma\right)$. We obtain

$$
\begin{aligned}
\frac{d}{d s}\left[\left(\alpha_{i}\right)_{k} \exp \left(k \int_{s}^{1} \Lambda_{i}\right)\right] & -\Lambda_{i} \exp \left(k \int_{s}^{1} \Lambda_{i}\right) \mathscr{B}_{i}(\alpha)_{k} \\
= & \frac{1}{\lambda_{i}-\lambda_{p}} \exp \left(k \int_{s}^{1} \Lambda_{i}\right)\left(F_{i}\right)_{k}, \text { for } i \leqslant p-1
\end{aligned}
$$

and

$$
\begin{aligned}
\frac{d}{d s}\left[\left(\alpha_{i}\right)_{k} \exp \left(k \int_{0}^{s} \Lambda_{i}\right)\right] & +\Lambda_{i} \exp \left(k \int_{0}^{s} \Lambda_{i}\right) \mathscr{B}_{i} \cdot \alpha_{k} \\
& =\frac{1}{\lambda_{i}-\lambda_{p}} \exp \left(k \int_{0}^{s} \Lambda_{i}\right)\left(F_{i}\right)_{k} \quad \text { for } i \geqslant p+1
\end{aligned}
$$

Integrating, we get

$$
\begin{aligned}
\left(\alpha_{i}\right)_{k}(s, y)= & \left(\alpha_{i}\right)_{k}(1, y) \cdot \exp \left(-k \int_{s}^{1} \Lambda_{i}\right) \\
& +\int_{s}^{1} \exp \left(-k \int_{s}^{s^{\prime}} \Lambda_{i}\right) \Lambda_{i} \mathscr{B}_{i} \cdot \alpha_{k} d s^{\prime} \\
& +\int_{s}^{1} \exp \left(-k \int_{s}^{s^{\prime}} \Lambda_{i}\right) \frac{1}{\lambda_{p}-\lambda_{i}}\left(F_{i}\right)_{k} d s^{\prime} \quad i \leqslant p-1, \\
\left(\alpha_{i}\right)_{k}(s, y)= & \left(\alpha_{i}\right)_{k}(0, y) \exp \left(-k \int_{0}^{s} \Lambda_{i}\right) \\
& +\int_{0}^{s} \exp \left(-k \int_{s^{\prime}}^{s} \Lambda_{i}\right) \Lambda_{i} \mathscr{B}_{i} \cdot \alpha_{k} d s^{\prime} \\
& +\int_{0}^{s} \exp \left(-k \int_{s^{\prime}}^{s} \Lambda_{i}\right) \frac{1}{\lambda_{i}-\lambda_{p}}\left(F_{i}\right)_{k} d s^{\prime}, \quad i \geqslant p+1 .
\end{aligned}
$$

It follows from (3.9) and (3.8a) that

$$
\begin{aligned}
\left|\left(\alpha_{i}\right)_{k}(s, y)\right| \leqslant & \left|\left(\alpha_{i}\right)_{k}(1, y)\right|+C_{0} \varepsilon_{*} \sup _{0 \leqslant s \leqslant 1}\left|\mathscr{B}_{i} \cdot \alpha_{k}\right| \\
& +\sup _{0 \leqslant s \leqslant 1}\left|\left(\mathscr{F}_{i}\right)_{k}\right|, \quad i \leqslant p-1, \\
\left|\left(\alpha_{i}\right)_{k}(s, y)\right| \leqslant & \left|\left(\alpha_{i}\right)_{k}(0, y)\right|+C_{0} \varepsilon_{*} \sup _{0 \leqslant s \leqslant 1}\left|\mathscr{B}_{i} \cdot \alpha_{k}\right| \\
& +\sup _{0 \leqslant s \leqslant 1}\left|\left(\mathscr{F}_{i}\right)_{k}\right|, \quad i \geqslant p+1,
\end{aligned}
$$

where

$$
\begin{aligned}
& \left(\mathscr{F}_{i}\right)_{k}=\int_{s}^{1} \exp \left(-k \int_{s}^{s^{\prime}} \Lambda_{i}\right) \frac{1}{\lambda_{p}-\lambda_{i}}\left(F_{i}\right)_{k} d s^{\prime}, \quad i \leqslant p-1, \\
& \left(\mathscr{F}_{i}\right)_{k}=\int_{0}^{s} \exp \left(-k \int_{s^{\prime}}^{s} \Lambda_{i}\right) \frac{1}{\lambda_{i}-\lambda_{p}}\left(F_{i}\right)_{k} d s^{\prime}, \quad i \geqslant p+1,
\end{aligned}
$$




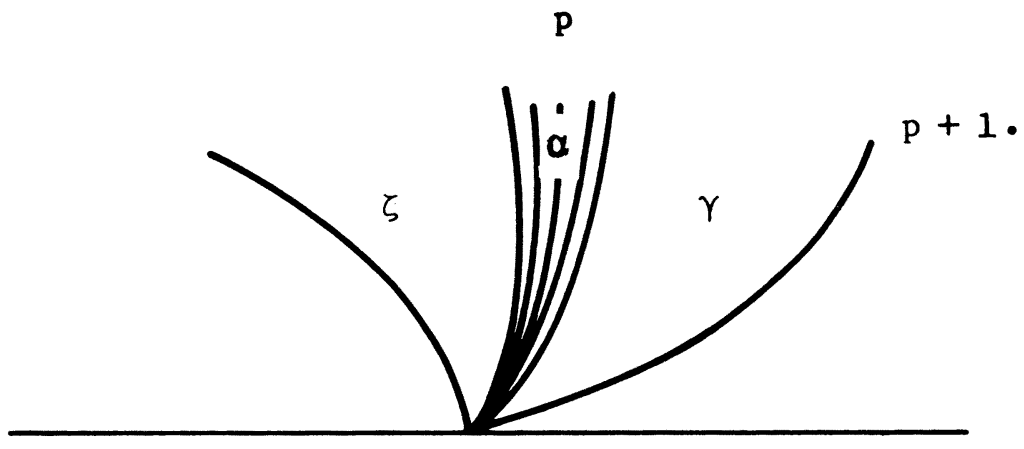

Figure 3.3

and since $\mathscr{B}_{i p}=0$ by $(2.20), \alpha_{k}=\left(\left(\alpha_{1}\right)_{k}, \ldots,\left(\alpha_{p-1}\right)_{k},\left(\alpha_{p+1}\right)_{k}, \ldots,\left(\alpha_{m}\right)_{k}\right)$. Now the boundary condition (2.6) gives us (see Figure 3.3 )

$$
\begin{array}{ll}
\left(\alpha_{i}\right)_{k}(1, y)=\left(\gamma_{i}\right)_{k 0}(y), & i \leqslant p-1, \\
\left(\alpha_{i}\right)_{k}(0, y)=\left(\zeta_{i}\right)_{0 k}(y), & i \geqslant p+1,
\end{array}
$$

with

$$
w_{m n}=\sum_{i}\left(\gamma_{i}\right)_{m n} r_{i}\left(w_{00}\right), \quad v_{m n}=\sum_{i}\left(\zeta_{i}\right)_{m n} r_{i}\left(v_{00}\right)
$$

where we have

$$
w_{00}=u_{0}(1, y), \quad v_{00}=u_{0}(0, y)
$$

by the continuity of the Riemann solution in rarefactions. Now

$$
\gamma_{k 0}(y), \quad i \leqslant p-1, \quad \zeta_{0 k}(y), \quad i \geqslant p+1,
$$

belong to our vector $\vec{b}_{k}$.

We will now adopt the convention that $C_{0}$ will denote a constant depending only on $u_{-}, f_{i}$, but it will get larger from equation to equation. With this, from (3.10) we obtain

$$
\left|\left(\alpha_{i}\right)_{k}(s, y)\right| \leqslant\left|\vec{b}_{k}\right|+C_{0} \varepsilon_{*} \sup _{s}\left|\alpha_{k}\right|+\sup _{s}\left|\mathscr{F}_{i k}\right|, \quad i \neq p
$$

For $\varepsilon_{*}<1 / 2 C_{0}$ we get by absorption

$$
\sup _{s}\left|\alpha_{k}\right| \leqslant 2\left|\vec{b}_{k}\right|+2 \sup _{s}\left|\left(\mathscr{F}_{k}\right)\right|
$$

where as before $\mathscr{F}_{k}=\left(\mathscr{F}_{1 k}, \mathscr{F}_{2 k}, \ldots\right)$. Using this in (3.10) we obtain

$$
\begin{array}{ll}
\left|\left(\alpha_{i}\right)_{k}(0, y)\right| \leqslant\left|\vec{b}_{k}\right|+C_{0} \varepsilon_{*}\left(2\left|\vec{b}_{k}\right|+2 \sup _{s}\left|\left(\mathscr{F}_{k}\right)\right|\right)+\sup _{s}\left|\left(\mathscr{F}_{i}\right)_{k}\right|, & i \leqslant p-1, \\
\left|\left(\alpha_{i}\right)_{k}(1, y)\right| \leqslant\left|\vec{b}_{k}\right|+C_{0} \varepsilon_{*}\left(2\left|\vec{b}_{k}\right|+2 \sup _{s}\left|\left(\mathscr{F}_{k}\right)\right|\right)+\sup _{s}\left|\left(\mathscr{F}_{i}\right)_{k}\right|, & i \geqslant p+1 .
\end{array}
$$


Now, by the boundary conditions,

$$
\begin{array}{ll}
\left(\alpha_{i}\right)_{k}(0, y)=\left(\zeta_{i}\right)_{0 k}, & i \leqslant p-1, \\
\left(\alpha_{i}\right)_{k}(1, y)=\left(\gamma_{i}\right)_{k 0}, & i \geqslant p+1,
\end{array}
$$

with $\zeta_{0, k}, i \leqslant p-1$, and $\gamma_{k, 0}, i \geqslant p+1$, belonging to $\vec{a}_{k}$. In fact, counting all rarefactions, they are the part of $\vec{a}_{k}$ on the faces of rarefactions boundaries. We call them $\vec{a}_{R}$. Hence we get

$$
\left|\left(\vec{a}_{R}\right)_{k}\right| \leqslant\left(1+2 C_{0} \varepsilon_{*}\right)\left|\vec{b}_{k}\right|+C_{0} \sup _{s}\left|\mathscr{F}_{k}\right| .
$$

(C) Estimates from shock, sound, and contact boundaries. The $\vec{a}_{k}$ 's occurring on the faces of shocks $\vec{a}_{S K}$, sound $\vec{a}_{S D}$, and contact surfaces $\vec{a}_{C}$ are handled by (2.24), (2.24a), (2.25b). From (2.24),

$$
\left|\left(\vec{a}_{S K}\right)_{k}\right| \leqslant\left|\left(\vec{b}_{S K}\right)_{k}\right|+O\left(\varepsilon_{*}\right)\left|S\left(\vec{a}_{S K}\right)_{k}\right|+O\left(\varepsilon_{*}\right)\left|T\left(\vec{b}_{S K}\right)_{k}\right|+\left|P(g)_{k}\right|
$$

which, for $\varepsilon_{*}$ small depending on $u_{-}, f_{i}$, implies that

$$
\left|\left(\vec{a}_{S K}\right)_{k}\right| \leqslant\left(1+O\left(\varepsilon_{*}\right)\right)\left|\left(\vec{b}_{S K}\right)_{k}\right|+C_{0}\left|(g)_{k}\right| .
$$

Similarly, from (2.25b),

$$
\left|\left(\vec{a}_{C}\right)_{k}\right| \leqslant\left(1+O\left(\varepsilon_{*}\right)\right)\left|\left(\vec{b}_{C}\right)_{k}\right|+C_{0}\left|(L)_{k}\right|,
$$

and from (2.24a),

$$
\left|\left(\vec{a}_{S D}\right)_{k}\right|=\left|\left(\vec{b}_{S D}\right)_{k}\right|
$$

This together with (3.11) gives us

$$
\left|\vec{a}_{k}\right| \leqslant\left(1+C_{0} \varepsilon_{*}\right)\left|\vec{b}_{k}\right|+C_{0}\left(\left|(g)_{k}\right|+\left|(L)_{k}\right|+\sup _{s}\left|\mathscr{F}_{k}\right|\right) \text {. }
$$

Combine this with (3.8) to get

$$
\begin{aligned}
\left|\vec{a}_{k}\right| \leqslant & \left(1+C_{0} \varepsilon_{*}\right) \rho^{k}\left|\vec{a}_{k}\right| \\
& +C_{0}\left(\left|\left(\alpha_{+}\right)_{k}\right|+\left|\left(\alpha_{-}\right)_{k}\right|+\left|\mathscr{F}_{k-1}\right|+\left|(g)_{k}\right|+\left|(L)_{k}\right|+\sup _{s}\left|\mathscr{F}_{k}\right|\right) .
\end{aligned}
$$

By choosing $\varepsilon_{*}$ smaller, but depending only on $u_{-}, f_{i}$, we can make $\left(1+C_{0} \varepsilon_{*}\right) \rho \leqslant \frac{1}{2}$. Hence the above together with (3.8) yield our main linear estimate

$$
\left|\begin{array}{l}
\vec{a}_{k} \\
\vec{b}_{k}
\end{array}\right| \leqslant C_{0}\left(\left|\left(\alpha_{+}\right)_{k}\right|+\left|\left(\alpha_{-}\right)_{k}\right|+\left|\mathscr{F}_{k-1}\right|+\left|g_{k}\right|+\left|(L)_{k}\right|+\sup _{0 \leqslant s \leqslant 1}\left|\mathscr{F}_{k}\right|\right)
$$

which holds for our choice of $\varepsilon_{*}$ and for $C_{0}$ depending on $u_{-}, f_{i}$ only. Now $\vec{a}_{k}, \vec{b}_{k}$ satisfy a linear system

$$
A\left(\begin{array}{l}
\vec{a}_{k} \\
b_{k}
\end{array}\right)=\mathscr{H}_{k}
$$

where $\mathscr{H}_{k}$ comprises of all the inhomogeneous terms $\left(\alpha_{+}\right)_{k},\left(\alpha_{-}\right)_{k}, \mathscr{F}_{k-1},(L)_{k}, g_{k}$, $\mathscr{F}_{k}$. The estimate (3.13) shows that the $2 m n \times 2 m n$ matrix $A$ is invertible. Formulas (3.1), (3.4), and (3.5) will give directly the rest of the coefficients in the gaps. Given 
the initial values $\left(\alpha_{i}\right)_{k}(0, y)$ we can solve the O.D.E. (2.19) for $0 \leqslant s \leqslant 1, i \neq p$. We can finally recover the rest of the unknowns, $\alpha_{p}$ in rarefactions and the surface coefficients, from the $p$ th equation at each boundary.

4. Convergence. In this section we prove the convergence of the power series constructed in the previous sections by employing a variant of the technique of majorization. To carry out this process we must consider our variables $s, y$ complex with

$$
\begin{aligned}
& y \in \Omega_{y}=\left\{y_{i} \in \mathbf{C}, d\left(y_{i},\left[-R_{0}, R_{0}\right]\right)<\delta, i=1, \ldots, d\right\}, \\
& s \in \Omega_{s}=\{s \in \mathbf{C}, d(s,[0,1])<\delta\} .
\end{aligned}
$$

REMARKS. $\delta$ is a small number less than 1 to be chosen later and $d(s,[0,1])$ represents the distance from $s$ to $[0,1]$.

If we begin with complex analytic initial data $u_{ \pm}$and complex analytic coefficients in our equation (1.2), it is clear that all our equations will hold for $y$ and $x$ compiex.

(A) Auxiliary lemmas. We define

$$
H_{k}=\left\{u(s, y) \text { analytic in } \Omega_{s} \times \Omega_{y}, \sup _{\substack{s \in \Omega_{s} \\ y \in \Omega_{y}}}\left(d\left(s, \Omega_{s}^{c}\right) \cdot d\left(s, \Omega_{y}^{c}\right)\right)^{k}|u(s, y)|<\infty\right\} \text {. }
$$

It follows that $H_{k}$ are Banach spaces with norm

$$
|u|_{H_{k}}=\sup _{\substack{s \in \Omega_{s} \\ y \in \Omega_{y}}}\left(d\left(s, \Omega_{s}^{c}\right) \cdot\left(s, \Omega_{y}^{c}\right)\right)^{k}|u(s, y)|
$$

We will use the notation $d_{s}=d\left(s, \Omega_{s}^{c}\right), d_{y}=d\left(y, \Omega_{y}^{c}\right)$ and note that $d_{s}, d_{y} \leqslant 1$ if $\delta \leqslant 1$. Hence $|u|_{H_{k+1}} \leqslant|u|_{H_{k}}$.

LEMMA 1 (HÖRMANDER [3, P. 117]).

$$
\left|u_{s}\right|_{H_{k+1}} \leqslant e(k+1)|u|_{H_{k}}, \quad\left|u_{y}\right|_{H_{k+1}} \leqslant e(k+1)|u|_{H_{k}},
$$

for $u \in H_{k}$.

Proof. It suffices to consider $u(s), s \in \Omega_{s}$, and show the first inequality. Fix $s \in \Omega_{s}$ and let $\varepsilon<d_{s}$. Then Cauchy's inequality gives

$$
\left|u^{\prime}(s)\right| \leqslant \varepsilon^{-1} \sup _{|\zeta-s| \leqslant \varepsilon}|u(\zeta)| \leqslant \varepsilon^{-1}\left(d_{s}-\varepsilon\right)^{-k}|u|_{H_{k}} .
$$

Choosing $\varepsilon=d_{s} /(k+1)$ we obtain

$$
\left|u^{\prime}(s)\right| \leqslant(k+1)\left(1+k^{-1}\right)^{k} d_{s}^{-k-1}|u|_{H_{k}} \leqslant(k+1) e d_{s}^{-k-1}|u|_{H_{k}} .
$$

The lemma results by multiplying through by $d_{s}^{k+1}$ and taking sup over $s \in \Omega_{s}$.

Lemma 2. Let $C>0$. Then there exists $\delta_{*}=\delta_{*}(C)$ such that

$$
e^{r C} \leqslant \delta /(\delta-r) \quad \text { for } 0 \leqslant r<\delta, \delta \leqslant \delta_{*} .
$$


Proof. Let $f(r)=e^{r C}-\delta /(\delta-r)$. Then

$$
f(0)=0 \text { and } f^{\prime}(r)=C e^{r C}-\delta /(\delta-r)^{2} .
$$

We have $C e^{r C} \leqslant C e^{\delta C} \leqslant 1 / \delta$ if $\delta<1 / C e=\delta_{*}(C)$, and since $1 / \delta<\delta /(\delta-r)^{2}$, $f^{\prime}(r)<0$ for $0 \leqslant r<\delta, \delta<\delta_{*}(C)$. Hence $f(r) \leqslant 0$ for $0 \leqslant r<\delta$.

LeMma 3. Given $N>0$, there exists $\delta_{*}(N)$ such that

$$
\ln \frac{\delta_{*}-r}{\delta_{*}-\rho} \leqslant-N(r-\rho), \quad 0 \leqslant \rho<r<\delta_{*} .
$$

Proof. Let $x+r-\rho$ and $\varepsilon=\delta_{*}-r$. It suffices to show

$$
\ln \left(\frac{1}{1+x / \varepsilon}\right) \leqslant-N x, \quad 0<x<\delta_{*}, 0<\varepsilon<\delta_{*},
$$

or $x^{-1} \ln (1+x / \varepsilon) \geqslant N$.

There exists $\delta_{0}(N)$ such that $x^{-1} \ln (1+(N+1) x) \geqslant N, 0<x \leqslant \delta_{0}$. Take $\delta_{*}=$ $\min \left(\delta_{0}, 1 /(N+1)\right)$. Then $x^{-1} \ln (1+x / \varepsilon)>x^{-1} \ln (1+(N+1) x)>N$ since $1 / \varepsilon$ $>1 / \delta_{*} \geqslant(N+1)$ and $x<\delta_{*} \leqslant \delta_{0}$.

\section{Lemma 4. Define}

$$
T_{i} u= \begin{cases}\int_{s}^{1} \exp \left(-k \int_{s}^{s^{\prime}} \Lambda_{i}(\sigma, y) d \sigma\right) u\left(s^{\prime}, y\right) d s^{\prime}, & i \leqslant p-1, \\ \int_{0}^{s} \exp \left(-k \int_{s^{\prime}}^{s} \Lambda_{i}(\sigma, y) d \sigma\right) u\left(s^{\prime}, y\right) d s^{\prime}, & i \geqslant p+1,\end{cases}
$$

for $u \in H_{k-1}, k \geqslant 1$.

Then there exists $\delta_{*}$, dependingly only on $u_{-}, f_{i}$, such that

$$
\begin{gathered}
\left|T_{i} u\right|_{H_{k-1}} \leqslant C_{0}|u|_{H_{k-1}}, \\
\left|T_{i} u\right|_{H_{k-1}} \leqslant(1 / k)\left(C_{0} / \varepsilon_{0}\right)|u|_{H_{k-1}},
\end{gathered}
$$

for $\delta \leqslant \delta_{*}$, with $C_{0}, \varepsilon_{0}$ as in (3.8a).

Proof. (3.8a) will hold for $s, y \in \Omega_{s} \times \Omega_{v}$ if $\delta$ is small depending only on $u_{-}, f_{i}$. It suffices to consider the $i \geqslant p+1$ case only. Fix $s \in \Omega_{s}$ and let $s^{*}$ be the point on $[0,1]$ closest to $s$. Let $r=|s-s *|$. (See Figure 4.1.)

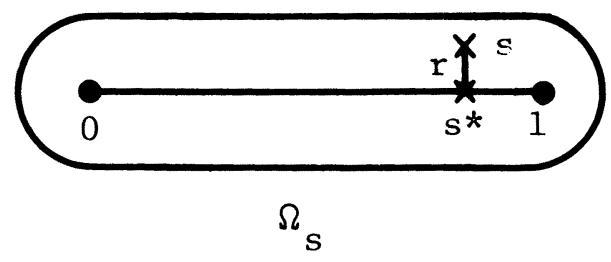

Figure 4.1 
Then if $s^{\prime} \in\left[0, s^{*}\right]$

$$
\begin{aligned}
\left|\exp \left(-k \int_{s^{\prime}}^{s} \Lambda_{i}\right)\right| & =\left|\exp \left(-k \int_{s^{\prime}}^{s^{*}} \Lambda_{i}\right)\right|\left|\exp \left(-k \int_{s^{*}}^{s} \Lambda_{i}\right)\right| \\
& \leqslant \exp \left(-k\left(s^{*}-s^{\prime}\right) \varepsilon_{0}\right) \exp \left(k r C_{0} \varepsilon_{*}\right) \text { by (3.8a). }
\end{aligned}
$$

Hence

$$
\begin{aligned}
I_{1} & =\left|\int_{0}^{s^{*}} \exp \left(-k \int_{s^{\prime}}^{s} \Lambda_{i}\right) u\left(s^{\prime}, y\right) d s^{\prime}\right| \\
& \leqslant \exp \left(k r C_{0} \varepsilon_{*}\right) \cdot \frac{|u|_{H_{k-1}}}{\left(\delta d_{y}\right)^{k-1}} \cdot \int_{0}^{s} \exp \left(-k\left(s^{*}-s^{\prime}\right) \varepsilon_{0}\right) d s^{\prime} \\
& =\frac{\exp \left(r C_{0} \varepsilon_{*}\right)}{d_{y}^{k-1}} \cdot\left(\frac{\exp \left(r C_{0} \varepsilon_{*}\right)}{\delta}\right)^{k-1} \frac{1}{k \varepsilon_{0}}\left(1-\exp \left(-k \varepsilon_{0} s^{*}\right)\right)|u|_{H^{k-1}}
\end{aligned}
$$

Applying Lemma 2, for $\delta<\delta_{*}\left(C_{0} \varepsilon_{*}\right)$ we get

$$
\leqslant \frac{\exp \left(\delta C_{0} \varepsilon_{*}\right)}{(\delta-r)^{k-1}} \frac{1}{d_{y}^{k-1}} \frac{1}{k \varepsilon_{0}}\left(1-\exp \left(-k \varepsilon_{0}\right)\right)|u|_{H^{k-1}} \quad \text { since } s^{*} \leqslant 1
$$

Next, let $s=s^{*}+r e^{i \theta}$ and $s^{\prime}=s^{*}+\rho e^{i \theta}$. Then

$$
\begin{aligned}
I_{2} & =\left|\int_{s^{*}}^{s} \exp \left(-k \int_{s^{\prime}}^{s} \Lambda_{i}\right) u\left(s^{\prime}, y\right) d s^{\prime}\right| \\
& \leqslant \int_{0}^{r} \exp \left(k(r-\rho) C_{0} \varepsilon_{*}\right) \cdot \frac{1}{(\delta-\rho)^{k-1}} d \rho \cdot \frac{|u|_{H_{k-1}}}{d_{y}^{k-1}} \\
& \leqslant \frac{\exp \left(r C_{0} \varepsilon_{*}\right)}{(\delta-r)^{k-1}} \frac{|u|_{H_{k-1}}}{d_{y}^{k-1}} \int_{0}^{r} \exp \left((k-1)\left[(r-\rho) C_{0} \varepsilon_{*}+\ln \left(\frac{\delta-r}{\delta-\rho}\right)\right]\right) d \rho .
\end{aligned}
$$

If we let $N=1+C_{0} \varepsilon_{*}$, the integral above equals

$$
\int_{0}^{r} e^{-(k-1)(r-\rho)} \exp \left((k-1)\left[(r-\rho) N+\ln \left(\frac{\delta-r}{\delta-\rho}\right)\right]\right) d \rho .
$$

Using Lemma 3 , for $\delta<\delta_{*}(N)$ we get

$$
\begin{aligned}
& \leqslant \int_{0}^{r} e^{-(k-1)(r-\rho)} d \rho= \begin{cases}(k-1)^{-1}\left(1-e^{-(k-1) r}\right), & k>1, \\
r, & k=1,\end{cases} \\
& \leqslant(e / k)\left(1-e^{-k r}\right) \text { for } k \geqslant 1
\end{aligned}
$$

since $r \leqslant 1$. Hence, for $\delta<\delta_{*}$ with $\delta_{*}$ depending on $u_{-}, f_{i}$ only,

$$
\left|T_{i} u(s)\right| \leqslant I_{1}+I_{2} \leqslant \frac{|u| H_{k-1}}{\left(d_{s} d_{y}\right)^{k-1}}\left[e^{\delta C_{0} \varepsilon_{*}} \cdot \frac{1}{k \varepsilon_{0}}\left(1-e^{-k \varepsilon_{0}}\right)+\frac{e}{k}\left(1-e^{-k \delta}\right)\right] .
$$

The inequality (4.3) follows immediately. The inequality (4.2) follows by observing that $x^{-1}\left(1-e^{-x C}\right) \leqslant C$ for $x, C>0$. 
(B) The majorant. Ultimately, we want to show that

$$
\begin{aligned}
& \left|\left(\alpha_{i}\right)_{k}\right|_{H_{k-1}} \leqslant \frac{1}{(k+1)^{2}} a_{k}, \\
& \left|\left(\alpha_{i}\right)_{m n}\right|_{H_{k-1}} \leqslant \frac{1}{(m+1)^{2}} \frac{1}{(n+1)^{2}}\left(\begin{array}{c}
m+n \\
n
\end{array}\right) a_{k}, \\
& \left|(k+1) \phi_{k+1}\right|_{H_{k-1}} \leqslant(k+1)^{-2} a_{k},
\end{aligned}
$$

for $i=1, \ldots, m, m+n=k, k \geqslant 1$.

Here $\phi$ denotes any boundary surface and $a(z)=a_{1} z+a_{2} z^{2}+\cdots, a_{j}>0$, will be a convergent power series. We are not yet ready to say what $a(z)$ is. The $a_{k}$ 's will satisfy a recursive relation which will be determined during the course of majorization.

REMARK. We define

$$
\left|\left(\alpha_{i}\right)_{m n}\right|_{H_{k-1}}=\sup _{y \in \Omega_{y}}\left(\delta d_{y}\right)^{k-1}\left|\left(\alpha_{i}\right)_{m n}(y)\right|
$$

where $\left(\alpha_{i}\right)_{m n}(y)$ is regarded is a function of $s$ and $y$ with $s=0$.

Let $a_{0}>0$ be an upper bound for all zero order coefficients $\left|\left(\alpha_{i}\right)_{0}(s, y)\right|$, $\left|\left(\alpha_{i}\right)_{00}(y)\right|, \quad\left|\phi_{1}(y)\right|=\left|\lambda_{\phi}(y)\right|$, as well as their derivatives $\left|\partial_{s}\left(\alpha_{i}\right)_{0}\right|,\left|\partial_{y}\left(\alpha_{i}\right)_{0}\right|$, $\left|\partial_{y}\left(\alpha_{i}\right)_{00}\right|,\left|\partial_{y} \phi_{1}\right|$ with $s, y \in \Omega_{s} \times \Omega_{y}$.

We now state the Main Majorization Lemma, which will be proved in (C), and use it for the rest of (B).

MAIn MAjorization Lemma. Suppose we have $a_{0}, a_{1}, \ldots, a_{k-1}, k \geqslant 1$ so that (4.4) is satisfied up to and including the index $k-1$. Then

$$
\begin{aligned}
& \left|\left(F_{i}\right)_{k}\right|_{H_{k-1}} \leqslant \frac{k+1}{(k+1)^{2}}\left(Q_{1}(a(z))+z R_{1}(a(z), z)\right)_{k}, \\
& \left|\left(F_{i}\right)_{m n}\right|_{H_{k-1}} \leqslant \frac{(m+n+1) !}{m ! n !} \frac{1}{(m+1)^{2}} \frac{1}{(n+1)^{2}}\left(Q_{2}+z R_{2}\right)_{k}, \\
& \left|\left(g_{i}\right)_{k}\right|_{H_{k-1}} \leqslant\left(Q_{3}+z R_{3}\right)_{k}, \\
& \left|\left(L_{i}\right)_{k}\right|_{H_{k-1}} \leqslant(k+1)^{-2}\left(Q_{4}+z R_{4}\right)_{k},
\end{aligned}
$$

with $\left(F_{i}\right)_{k},\left(F_{i}\right)_{m n},\left(g_{i}\right)_{k},\left(L_{i}\right)_{k}$ from (2.17), (2.12), (2.22), (2.25a) and (2.29) respectively.

In (4.5) $Q_{j}(a)=Q_{j 2} a^{2}+Q_{j 3} a^{3}+\cdots, j=1,2,3,4$ is a convergent power series beginning with quadratic terms and $R_{j}(a, z)$ is analytic at $(0,0)$ bearing no relation to the Riemann invariants introduced before.

REMARK. $Q_{j}, R_{j}$ will, as expected, involve majorants of the coefficients $A, B, L, \ldots$ of the original equation. We note that $\left(Q_{1}+z R_{1}\right)+\left(Q_{2}+z R_{2}\right)=\left(Q_{1}+Q_{2}\right)+$ $z\left(R_{1}+R_{2}\right)$. Hence sums of functions of this form have the same form, and we will simply denote them all by $Q+z R$ in spite of the fact that they may differ from equation to equation. 
Assuming the lemma, we now prove (4.4) for the index $k$.

We consider $\left(\mathscr{F}_{i}\right)_{m n}$ from (3.1a), (3.1b). For $i \leqslant p$, by (4.5)

$$
\begin{aligned}
& \rho_{i}^{j} \frac{(n+1)(n+2) \cdots(n+j)}{(m+1) \cdot m \cdots(m-j+1)} \frac{1}{\left|\lambda_{\psi}-\lambda_{i}\right|}\left|\left(F_{i}\right)_{m-j, n+j}\right|_{H_{k-1}} \\
& \leqslant \rho_{i}^{j} C_{0} \frac{(n+1) \cdots(n+j)}{(m+1) \cdots(m-j+1)} \cdot \frac{(m+n+1) !}{(m-j) !(n+j) !} \\
& \cdot(m-j+1)^{-2}(n+j+1)^{-2} \cdot(Q+z R)_{k} \\
& =C_{0}\left(\begin{array}{c}
m+n+1 \\
n
\end{array}\right)(m+1)^{-2}(n+1)^{-2} \rho_{i_{1}}^{j}(Q+z R)_{k} \\
& \cdot \rho_{i_{2}}^{j} \frac{(m+1)^{2}(n+1)^{2}}{(m-j+1)^{2}(n+j+1)^{2}}
\end{aligned}
$$

where $\rho_{i}=\rho_{i_{1}} \cdot \rho_{i_{2}}$ and $\rho_{i_{1}}, \rho_{i_{2}}<1$. The expression

$$
\rho_{i_{2}}^{j} \frac{(m+1)^{2}(n+1)^{2}}{(m-j+1)^{2}(n+j+1)^{2}}
$$

is bounded independent of $m, n, j$ since we write it as

$$
\rho_{i_{2}}^{j}(j+1)^{2} \frac{(m+1)^{2}(n+1)^{2}}{(j+1)^{2}(m-j+1)^{2}(n+j+1)^{2}} \text { and } \frac{(m+1)^{2}}{(j+1)^{2}(m-j+1)^{2}}
$$

is bounded by 4 , by considering $j<m / 2$ and $j>m / 2$. This gives

$$
\begin{aligned}
\left|\left(\mathscr{F}_{i}\right)_{m n}\right|_{H_{k-1}} \leqslant & \frac{C_{0}}{(m+1)^{2}(n+1)^{2}}\left(\begin{array}{c}
m+n+1 \\
n
\end{array}\right)(Q+z R)_{k} \\
& \cdot\left(1+\rho_{i_{1}}+\rho_{i_{1}}^{2}+\cdots \rho_{i_{1}}^{n}\right) \\
\leqslant & \frac{C_{0}}{(m+1)^{2}(n+1)^{2}}\left(\begin{array}{c}
m+n+1 \\
n
\end{array}\right)(Q+z R)_{k} \quad \text { for } i \leqslant \rho .
\end{aligned}
$$

Similarly,

$$
\left|\left(\mathscr{F}_{i}\right)_{m n}\right|_{H_{k-1}} \leqslant \frac{C_{0}}{(m+1)^{2}(n+1)^{2}}\left(\begin{array}{c}
m+n+1 \\
n
\end{array}\right)(Q+z R)_{k} \quad \text { for } i \geqslant p+1 .
$$

REMARK. In all of the above, $m+n+1=k$.

We now estimate $\left(\mathscr{F}_{i}\right)_{k}$ 's in rarefactions from their formulas given after (3.10).

Using the first estimate in (4.5) and (4.3) of Lemma 4,

$$
\left|\left(\mathscr{F}_{i}\right)_{k}\right|_{H_{k-1}} \leqslant \frac{C_{0}}{\varepsilon_{0}} \frac{1}{(k+1)^{2}}(Q+z R)_{k} .
$$

We are now ready to get an estimate on $\vec{a}_{k}, \vec{b}_{k}$ from (3.13).

For $k \geqslant 1$, since $\alpha_{ \pm}$is analytic,

$$
\left|\left(\alpha_{ \pm}\right)_{k}(y)\right| \leqslant A_{0} C^{k-1} \leqslant A_{0} \frac{1}{(k+1)^{2}} \frac{C_{1}^{k-1}}{\left(\delta d_{y}\right)^{k-1}}
$$


where $A_{0}, C, C_{1}$ are appropriately chosen and $C<C_{1}$. Hence

$$
\left|\left(\alpha_{ \pm}\right)_{k}\right|_{H_{k-1}} \leqslant(k+1)^{-2}(z R(z))_{k}
$$

for $R(z)=A_{0} \sum_{i \geqslant 0} C_{1}^{j} z^{i}$ analytic at $z=0$. Also (4.8) implies

$$
\delta^{k-1} d_{y}^{k-1} \sup _{0 \leqslant s \leqslant 1}\left|\mathscr{F}_{k}\right| \leqslant \frac{C_{0}}{\varepsilon_{0}} \frac{1}{(k+1)^{2}}(Q+z R)_{k} .
$$

This, together with (4.5) -(4.7), applied to the right-hand side of (3.13) implies

$$
\left|\left(\vec{b}_{i}\right)_{k}\right|_{H_{k-1}},\left|\left(\vec{a}_{i}\right)_{k}\right|_{H_{k-1}} \leqslant C(k+1)^{-2}(Q+z R)_{k}
$$

with $C$ depending on $u_{-}, f_{i}$ as well as $\varepsilon_{0}$.

To estimate the remaining coefficients in the gaps we consider (3.1c). We get, by using (4.9),

$$
\left|\rho_{i}^{m+1}\left(\alpha_{i}\right)_{0 k}\right|_{H_{k-1}} \leqslant \rho_{i}^{m+1} \frac{(m+1)^{2}(n+1)^{2}}{(m+n+1)^{2}} \cdot \frac{C}{(m+1)^{2}(n+1)^{2}}(Q+z R)_{k} .
$$

Letting $C$, which depends on $\varepsilon_{0}, u_{-}, f_{i}$, get larger from equation to equation, as we did with $C_{0}$, we get

$$
\leqslant C(m+1)^{-2}(n+1)^{-2}(Q+z R)_{k} .
$$

Using (4.6) as well, we obtain from (3.1c)

(4.10) $\left|\left(\alpha_{i}\right)_{m+1, n}\right|_{H_{k-1}} \leqslant \frac{C}{(m+1)^{2}(n+1)^{2}}\left(\begin{array}{c}m+n+1 \\ n\end{array}\right)(Q+z R)_{k}$ for $i \leqslant p$.

Similarly,

$$
\left|\left(\alpha_{i}\right)_{m, n+1}\right|_{H_{k-1}} \leqslant \frac{C}{(m+1)^{2}(n+1)^{2}}\left(\begin{array}{c}
m+n+1 \\
m
\end{array}\right)(Q+z R)_{k} \quad \text { for } i \geqslant p+1 .
$$

Remarks. Formula (4.10) holds for the $(m+1)$ th gap too, and $(4.11)$ holds for the 1st gap (see (3.4) and (3.5)).

To get estimates for the rarefaction coefficients we consider (3.9) with $(s, y) \in \Omega_{s}$ $\times \Omega_{y}$. Letting $H_{k-1}^{n-1}=H_{k-1} \times \cdots \times H_{k-1},(n-1)$ times, we introduce the map $\mathscr{T}$ defined by

$$
\left(\mathscr{T}_{v}\right)_{i}=\left\{\begin{array}{cc}
\left(\alpha_{i}\right)_{k}(1, y) \exp \left(-k \int_{s}^{1} \Lambda_{i}\right)+T_{i}\left(\Lambda_{i} \mathscr{B}_{i} \cdot v\right) \\
+T_{i}\left(\frac{1}{\lambda_{p}-\lambda_{i}}\left(F_{i}\right)_{k}\right), \quad i \leqslant p-1, \\
\left(\alpha_{i}\right)_{k}(0, y) \exp \left(-k \int_{0}^{s} \Lambda_{i}\right)+T_{i}\left(\Lambda_{i} \mathscr{B}_{i} \cdot v\right) \\
+T_{i}\left(\frac{1}{\lambda_{p}-\lambda_{i}}\left(F_{i}\right)_{k}\right), \quad i \geqslant p+1,
\end{array}\right.
$$

for $v \in H_{k-1}^{n-1}$. 
REMARKS. Since in (2.20), $\mathscr{B}_{i, p}=0$, in the formulas above,

$$
\mathscr{B}_{i}=\left(\mathscr{B}_{i, 1}, \ldots, \mathscr{B}_{i, p-1}, \mathscr{B}_{i, p+1} \cdots \mathscr{B}_{i, m}\right) \in \mathbf{R}^{n-1}
$$

$T_{i}$ 's are the maps defined in Lemma 4. Note that (3.9) means $\mathscr{T}(\alpha)_{k}=(\alpha)_{k}$. We want to show that $\mathscr{T}$ is a contraction mapping some ball in $H_{k-1}^{n-1}$ to itself. This will give us a fixed point in the ball.

Using (4.3a) with $s^{\prime}=0$, and (4.9) we obtain

$$
\left|\left(\alpha_{i}\right)_{k}(0, y) \exp \left(-k \int_{0}^{s} \Lambda_{i}\right)\right| \leqslant \frac{C}{(k+1)^{2}}(Q+z R)_{k} \cdot \frac{1}{d_{y}^{k-1}}\left(\frac{e^{r C_{0} \varepsilon_{*}}}{\delta}\right)^{k-1} e^{r C_{0} \varepsilon_{*}} \text {. }
$$

Applying Lemma 2 we obtain

$$
\left|\alpha_{i}(0, y) \exp \left(-k \int_{0}^{s} \Lambda_{i}\right)\right|_{H_{k-1}} \leqslant \frac{C}{(k+1)^{2}}(Q+z R)_{k} .
$$

Similarly,

$$
\left|\alpha_{i}(1, y) \exp \left(-k \int_{s}^{1} \Lambda_{i}\right)\right|_{H_{k-1}} \leqslant \frac{C}{(k+1)^{2}}(Q+z R)_{k} .
$$

Since

$$
\left|\Lambda_{i} \mathscr{B}_{i} \cdot \alpha_{k}\right|_{H_{k-1}} \leqslant C_{0} \varepsilon_{*}\left|\alpha_{k}\right|_{H_{k-1}^{n-1}}
$$

by (4.2),

$$
\left|T_{i}\left(\Lambda_{i} \mathscr{B} \cdot \alpha_{k}\right)\right|_{H_{k-1}} \leqslant C_{0} \varepsilon_{*}\left|\alpha_{k}\right|_{H_{k-1}^{n-1}}
$$

As always, $C_{0}$ depends only on $u_{-}, f_{i}$. We choose $\varepsilon_{*}$ small enough so that $C_{0} \varepsilon_{*}<1$. Using the first inequality in (4.5) and (4.3), we get

$$
\left|T_{i}\left(\frac{1}{\lambda_{p}-\lambda_{i}}\left(F_{i}\right)_{k}\right)\right|_{H_{k-1}} \leqslant \frac{C_{0}}{\varepsilon_{0}(k+1)^{2}}(Q+z R)_{k} .
$$

In conclusion, adding all the estimates above,

$$
\left|\mathscr{T}(\alpha)_{k}\right|_{H_{k-1}^{n-1}}=\max _{i}\left|\left(\mathscr{T} \alpha_{k}\right)_{i}\right|_{H_{k-1}} \leqslant \frac{C}{(k+1)^{2}}(Q+z R)_{k}+C_{0} \varepsilon_{*}\left|(\alpha)_{k}\right|_{H_{k-1}^{n-1}}
$$

Choose $D$ so that $C+C_{0} \varepsilon_{*} D \leqslant D$, which is possible since $C_{0} \varepsilon_{*}<1$. Thus

$$
\left|\mathscr{T}(\alpha)_{k}\right|_{H_{k-1}^{n-1}} \leqslant D(k+1)^{-2}(Q+z R)_{k}
$$

if

$$
\left|\left(\alpha_{k}\right)\right|_{H_{k-1}^{n-1}} \leqslant D(k+1)^{-2}(Q+z R)_{k}
$$

Now

$$
\left|(\mathscr{T} v)_{i}-(\mathscr{T} u)_{i}\right|_{H_{k-1}}=\left|T_{i}\left(\Lambda_{i} \mathscr{B}_{i} \cdot(v-u)\right)\right|_{H_{k-1}} \leqslant C_{0} \varepsilon_{*}|v-u|_{H_{k-1}^{n-1}}
$$

with $C_{0} \varepsilon_{*}<1$ as in (4.13a). Hence $\mathscr{T}$ is a contraction, which has a fixed point $(\alpha)_{k}$ satisfying

$$
\left|\alpha_{k}\right|_{H_{k-1}^{n-1}} \leqslant D(k+1)^{-2}(Q+z R)_{k} .
$$


Remarks. $D$ tends to infinity as $\varepsilon_{0}$ tends to zero since $C$ does, which means that the radius of convergence of our series approaches zero as rarefactions degenerate.

In (4.14), $(\alpha)_{k}=\left(\left(\alpha_{1}\right)_{k}, \ldots,\left(\alpha_{p-1}\right)_{k},\left(\alpha_{p+1}\right)_{k}, \ldots,\left(\alpha_{m}\right)_{k}\right)$. As a fixed point of $\mathscr{T}$, $\alpha_{k}$ is the solution to the rarefaction O.D.E.'s (2.19). In $\$ 3$ we could have obtained the existence of $(\alpha)_{k}$ by solving the initial value O.D.E. in the complex domain. However, in this chapter we were able to obtain the estimate (4.14) in addition to the existence.

Although the rarefaction surface coefficients could be obtained from (2.20), we cannot prove the desired estimate on them from the equation because of the $(k+1)$ factor in front of the $\left(\alpha_{p}\right)_{k}$ term. Formula (2.20) is not adequate for bounding derivatives of $\phi$ or $\psi$. Fortunately, we have (2.25), (2.28) which were derived as a consequence of $\phi$ being characteristic.

From (2.28), using (4.9) and (4.5) we obtain

$$
\left|(k+1) \phi_{k+1}\right|_{H_{k-1}} \leqslant C(k+1)^{-2}(Q+z R)_{k}
$$

which holds for rarefaction and sound surfaces. By (2.25c), using (4.9) and (4.5) again, it clearly holds for contact surfaces as well.

We now go back to (2.20) to obtain the estimate on $\alpha_{p}(s, y)$. We use (4.5) to bound $\left(F_{p}\right)_{k}$ and (4.14), (4.15) to obtain

$$
\left|\left(\alpha_{p}\right)_{k}\right|_{H_{k-1}} \leqslant C(k+1)^{-2}(Q+z R)_{k} .
$$

REMARKS. To get (4.16) we needed to estimate

$$
\begin{aligned}
& \sup _{\substack{s \in \Omega_{s} \\
y \in \Omega_{y}}}\left|\left(d_{s} d_{y}\right)^{k-1}\right|\left|\phi_{k+1}+s\left(\psi_{k+1}-\phi_{k+1}\right)\right| \\
& \quad \leqslant \sup _{\substack{s \in \Omega_{s} \\
y \in \Omega_{y}}}\left(\delta d_{y}\right)^{k-1}\left|\phi_{k+1}-s\left(\psi_{k+1}-\phi_{k+1}\right)\right| \leqslant \frac{C}{(k+1)^{2}}(Q+z R)_{k} \text { by (4.15). }
\end{aligned}
$$

Finally, the shock surfaces coefficients from (2.23) satisfy

$$
\left|(k+1) \phi_{k+1}\right|_{H_{k-1}} \leqslant C(k+1)^{-2}(Q+z R)_{k} .
$$

ReMARKs. To get (4.17) we used (4.5) to bound $\left(g_{p}\right)_{k}$ and (4.9) to bound $(\alpha)_{k 0}$, $(\beta)_{0 k}$ from (2.23). Also, $1 /\left(\varepsilon_{p}+O\left(\varepsilon_{p}^{2}\right)\right) \leqslant C_{0} / \varepsilon_{0}$ is incorporated into the constant $C$.

Consider the sum of all $C(Q+z R)_{k}$ 's from (4.10), (4.11), and (4.14)-(4.17) and call it $Q+z R$ as agreed. Now set

$$
a_{k}=(Q(a(z))+z R(a(z), z))_{k}, \quad k \geqslant 1 .
$$

REMARKS. $(Q+z R)_{k}$ in (4.18) contains coefficients of $a$ of order less than $k$. By the implicit function theorem the equations $a(0)=0, a=Q(a)+z R(a, z)$ have a unique analytic solution $a(z)$ whose coefficients satisfy (4.18).

With this definition of $a_{k},(4.10),(4.11),(4.14)-(4.16)$ imply (4.4) for the index $k$.

Assuming the Main Majorization Lemma we have thus proved (4.4) for all $k \geqslant 1$. 
RemarKs. When $k=1$ the Main Majorization Lemma implies (4.5) with $Q_{i} \equiv 0$ and $R_{i}$ depending on $a_{0}$ only.

(C) Proof of the Main Majorization Lemma.

\section{LEMMA 5.}

$$
\sum_{\mu=0}^{m} \frac{1}{(m-\mu+1)^{2}} \cdot \frac{1}{(\mu+1)^{2}} \leqslant \frac{K_{0}}{(m+1)^{2}}, \quad m \geqslant 0,
$$

with $K_{0}$ a fixed numerical constant.

Proof. We let $[x]$ be the integer part of $x$. Then

$$
\begin{aligned}
\sum_{\mu=0}^{m} \frac{1}{(m-\mu+1)^{2}} & \frac{1}{(\mu+1)^{2}} \leqslant 2 \sum_{\mu=0}^{[m / 2]} \frac{1}{(m-\mu+1)^{2}(\mu+1)^{2}} \quad \text { (by symmetry) } \\
& \leqslant \frac{2}{(m-[m / 2]+1)^{2}} \sum_{\mu=0}^{\infty} \frac{1}{(\mu+1)^{2}} \leqslant \frac{8}{(m+1)^{2}} \sum_{\mu=0}^{\infty} \frac{1}{(\mu+1)^{2}} .
\end{aligned}
$$

LEMMA 6.

$$
\left(\begin{array}{c}
m_{1} \\
n_{1}
\end{array}\right)\left(\begin{array}{c}
m_{2} \\
n_{2}
\end{array}\right) \leqslant\left(\begin{array}{c}
m_{1}+m_{2} \\
n_{1}+n_{2}
\end{array}\right) .
$$

Proof. Consider $m_{1}+m_{2}$ objects. Then the left-hand side represents the number of ways we can choose $n_{1}$ objects out of the first $m_{1}$ and $n_{2}$ out of the remaining $m_{2}$. The right-hand side represents the number of ways we can choose $n_{1}+n_{2}$ out of $m_{1}+m_{2}$ with no restrictions. Hence, the inequality in Lemma 6 becomes evident.

Lemmas 5 and 6 are among the tools used for a proof of the Cauchy-Kovalevsky Theorem in [4].

LeMma 7. Let

$$
\begin{aligned}
u(\xi, \eta, y) & =\sum_{\substack{\mu \geqslant 0 \\
\nu \geqslant 0}} u_{\mu, \nu}(y) \xi^{\mu} \eta^{\nu}, \quad v(\xi, \eta, y)=\sum_{\substack{\mu \geqslant 0 \\
\nu \geqslant 0}} v_{\mu, \nu}(y) \xi^{\mu} \eta^{\nu} \\
w(\xi, \eta, y) & =\sum_{\substack{\mu \geqslant 0 \\
\nu \geqslant 0}} w_{\mu, \nu}(y) \xi^{\mu} \eta^{\nu}
\end{aligned}
$$

and suppose that for positive constants $T_{i}, i=0, \ldots, 2$, we have

$$
\begin{aligned}
&\left|u_{\mu, \nu}\right|_{H_{\mu+\nu-1}} \leqslant \frac{T_{0}}{(\mu+1)^{2}} \frac{1}{(\nu+1)^{2}}\left(\begin{array}{c}
\mu+\nu \\
\nu
\end{array}\right) a_{\mu+\nu}, \quad 1 \leqslant \mu+\nu \leqslant m+n, \\
&\left|w_{\mu, \nu}\right|_{H_{\mu+\nu-1}} \leqslant \frac{T_{1}}{(\mu+1)^{2}} \frac{1}{(\nu+1)^{2}}\left(\begin{array}{c}
\mu+\nu \\
\nu
\end{array}\right) b_{\mu+\nu}, \quad 1 \leqslant \mu+\nu \leqslant m+n, \\
&\left|v_{\mu, \nu}\right|_{H_{\mu+\nu}} \leqslant \frac{T_{2}}{(\mu+1)^{2}(\nu+1)^{2}} \frac{(\mu+\nu+1) !}{\mu ! \nu !} c_{\mu-\nu}, \quad 0 \leqslant \mu+\nu \leqslant m+n,
\end{aligned}
$$


with

$$
\begin{aligned}
& a(z)=\sum_{i \geqslant 1} a_{i} z^{i}, \quad a_{i} \geqslant 0, \quad b(z)=\sum_{i \geqslant 1} b_{i} z^{i}, \quad b_{i} \geqslant 0, \\
& c(z)=\sum_{i \geqslant 0} c_{i} z^{i}, \quad c_{i} \geqslant 0 .
\end{aligned}
$$

If $\left|u_{00}\right|_{H_{0}},\left|w_{00}\right|_{H_{0}}$ are also bounded by $T_{0} a_{0}, T_{1} b_{0}$ respectively, $a_{0}, b_{0} \geqslant 0$, then (4.19) $\left|(u \cdot w)_{m n}\right|_{H_{m+n-1}}$

$$
\leqslant \frac{K_{0}^{2} T_{0} T_{1}}{(m+1)^{2}(n+1)^{2}}\left(\begin{array}{c}
m+n \\
n
\end{array}\right)\left(\left(a(z)+a_{0}\right)\left(b(z)+b_{0}\right)\right)_{m+n},
$$

with $m+n \geqslant 1$.

(4.19a) In case $u_{00}=0=a_{0}$ or $w_{00}=0=b_{0}$ then we get (4.19) under the weaker hypothesis that $w_{\mu \nu}, u_{\mu \nu}$, respectively, satisfy their estimates for $1 \leqslant \mu+\nu \leqslant m+n-$ 1 only.

$$
\begin{aligned}
&\left|(u \cdot v)_{m n}\right|_{H_{m+n}} \leqslant \frac{K_{0}^{2} T_{0} T_{1}}{(m+1)^{2}(n+1)^{2}} \frac{(m+n+1) !}{m ! n !}\left[\left(a(z)+a_{0}\right) c(z)\right]_{m+n}, \\
& m+n \geqslant 0 .
\end{aligned}
$$

(4.20a) In case $u_{00}=0=a_{0}$ we get (4.20) with $\left|(u \cdot v)_{m n}\right|_{H_{m+n-1}}$ as the left-hand side, $m+n \geqslant 1$, under the weaker hypothesis that $v_{\mu, \nu}$ satisfies the estimate for $0 \leqslant \mu+\nu \leqslant m+n-1$ only. If, in addition, $v_{00}=0=c_{0}$, the hypothesis on $u_{\mu, \nu}$ could be weakened to $1 \leqslant \mu+\nu \leqslant m+n-1$.

Proof. We have

$$
\begin{aligned}
& \left|\left(d_{y} \delta\right)^{m+n-1}(u w)_{m n}\right| \leqslant\left(d_{y} \delta\right)^{m+n-1}\left|u_{m n}\right|\left|w_{00}\right|+\left(d_{y} \delta\right)^{m+n-1}\left|u_{00}\right|\left|w_{m n}\right| \\
& +\sum_{\substack{\mu=0 \\
\mu+\nu \neq 0, m+n}}^{m} \sum_{\substack{\nu=0 \\
\mu \nu}}^{n}\left|\left(d_{y} \delta\right)^{\mu+\nu-1}\right| w_{m-\nu, n-\nu} \mid\left(d_{y} \delta\right)^{m+n-(\mu+\nu)-1}\left(d_{y} \delta\right) \\
& \leqslant T_{0} a_{0}\left|w_{m, n}\right|_{H_{m+n-1}}+T_{1} b_{0}\left|u_{m, n}\right|_{H_{m+n-1}} \\
& +\sum_{\substack{\mu, \nu \\
\mu+\nu \neq 0, m+n}}\left|u_{\mu, \nu}\right|_{H_{\mu+\nu-1}}\left|w_{m-\mu, n-\nu}\right|_{H_{m+n-(\mu-\nu)-1}} \\
& \leqslant \frac{T_{0} T_{1}}{(m+1)^{2}(n+1)^{2}}\left(\begin{array}{c}
m+n \\
n
\end{array}\right)\left[a_{0} b_{m+n}+b_{0} a_{m+n}\right] \\
& +T_{0} T_{1} \sum_{\substack{\mu, \nu \\
\mu+\nu \neq 0, m+n}}\left(\begin{array}{c}
\mu+\nu \\
\nu
\end{array}\right)\left(\begin{array}{c}
m+n-(\mu+\nu-\nu) \\
n-\nu
\end{array}\right) \\
& \frac{1}{(\mu+1)^{2}} \frac{1}{(m-\mu+1)^{2}} \frac{1}{(\nu+1)^{2}} \cdot \frac{1}{(n-\nu+1)^{2}} a_{\mu+\nu} b_{m+n-(\mu+\nu)} .
\end{aligned}
$$


The second term above is

$$
\begin{aligned}
\leqslant & T_{0} T_{1} \sum_{l=1}^{m+n-1}\left(\begin{array}{c}
m+n \\
n
\end{array}\right) a_{l} b_{m+n-l}\left(\sum_{\mu=0}^{m} \frac{1}{(\mu+1)^{2}} \frac{1}{(m-\mu+1)^{2}}\right) \\
& \cdot\left(\sum_{\nu=0}^{n} \frac{1}{(\nu+1)^{2}} \frac{1}{(n-\nu+1)^{2}}\right) \\
\leqslant & \frac{T_{0} T_{1} K_{0}^{2}}{(m+1)^{2}(n+1)^{2}}\left(\begin{array}{c}
m+n \\
n
\end{array}\right) \sum_{l=1}^{m+n-1} a_{l} b_{m+n-l} .
\end{aligned}
$$

The estimate (4.19) follows. The result in (4.19a) follows from the fact that the right-hand side of (4.21) will not have a $w_{m n}$ or $u_{m n}$ term in case $u_{00}$, respectively $w_{00}$, is zero.

In case $m=n=0,(4.20)$ follows immediately. Hence, we assume $m+n \geqslant 1$. Since $d_{y}<1$,

$$
\begin{aligned}
\mid\left(d_{y} \delta\right)^{m+n} & (u \cdot v)_{m, n}\left|\leqslant\left(d_{y} \delta\right)^{m+n-1}\right| u_{m, n}|| v_{0,0}\left|+\left(d_{y} \delta\right)^{m+n}\right| v_{m, n}|| u_{00} \mid \\
& +\sum_{\substack{\mu, \nu \\
\mu+\nu \neq 0, m+n}}\left|u_{\mu, \nu}\right|\left(d_{y} \delta\right)^{\mu+\nu-1}\left|v_{m-\mu, n-\nu}\right|\left(d_{y} \delta\right)^{m+n-(\mu+\nu)} \\
\leqslant & \left|u_{m, n}\right|_{H_{m+n-1}} T_{2} c_{0}+\left|v_{m, n}\right|_{H_{m+n}} T_{0} a_{0} \\
& +\sum_{\substack{\mu, \nu \\
\mu \neq 0, m+n}}\left|u_{\mu, \nu}\right|_{H_{\mu+\nu-1}}\left|v_{m-\mu, n-\nu}\right|_{H_{m+n-(\mu+\nu)}} \\
\leqslant & \frac{T_{0} T_{2}}{(m+1)^{2}} \frac{1}{(n+1)^{2}} \frac{(m+n+1) !}{m ! n !}\left[c_{0} a_{m+n}+c_{m+n} a_{0}\right] \\
& +T_{0} T_{2} \sum_{\substack{\mu, \nu \\
(m+\nu \neq 0, m+n}}\left(\begin{array}{c}
\mu+\nu \\
\nu
\end{array}\right)(m+n-(\mu+\nu)+1) \cdot(m-\mu+1) \\
& \cdot \frac{1}{(\mu+1)^{2}(m-\mu+1)^{2}(\nu+1)^{2}(n-\nu+1)^{2}} a_{\mu+\nu} c_{m+n-(\mu-\nu)} .
\end{aligned}
$$

Since

$$
\begin{aligned}
\left(\begin{array}{c}
\mu+\nu \\
\nu
\end{array}\right)\left(\begin{array}{c}
m+n-(\mu+\nu)+1 \\
n-\nu
\end{array}\right)(m-\mu+1) & \leqslant\left(\begin{array}{c}
m+n+1 \\
n
\end{array}\right)(m+1) \\
& =\frac{(m+n+1) !}{m ! n !}
\end{aligned}
$$

(4.20) follows. The result in (4.20a) follows since the right-hand side of (4.22) will not have the $\left(d_{y} \delta\right)^{m+n} v_{m, n} u_{00}$ term in case $a_{0}=0$. Therefore, (4.22) will be valid with $\left|\left(d_{y} \delta\right)^{m+n-1}(u \cdot v)_{m n}\right|$ as left-hand side. The rest of (4.20a) is immediate.

COROLlaRY 1. Let $u_{i}=\sum\left(u_{i}\right)_{\mu \nu}(y) \xi^{\mu} \eta^{\nu}, i=1, \ldots, n$, satisfy the hypothesis of $u$ in Lemma 7. Then, for $\alpha=\left(\alpha_{1}, \ldots, \alpha_{n}\right)$, a multi-index

$$
\left|\left(u^{\alpha}\right)_{m, n}\right|_{H_{m+n-1}} \leqslant \frac{\left(K_{0} T_{0}\right)^{|\alpha|}}{(m+1)^{2}(n+1)^{2}}\left(\begin{array}{c}
m+n \\
n
\end{array}\right)\left(\left(a+a_{0}\right)^{|\alpha|}\right)_{m+n} .
$$


Proof. Follows from Lemma 7 (4.19) by induction on $|\alpha|$.

COROllary 2. Let $u=\left(u_{1}, \ldots, u_{n}\right)$ as in Corollary 1. Let $a(u, y)=$ $\sum a_{\alpha}\left(u_{00}, y\right)\left(u-u_{00}\right)^{\alpha}$ be analytic in the variables $u, y \in\left\{\left|u-u_{00}\right|<\varepsilon\right\} \times \Omega_{y}$. Suppose, by letting $u_{00}(y)=u(0,0, y)$, we have

$$
\sum_{|\alpha|=l}\left|a_{\alpha}\left(u_{00}(y), y\right)\right| \leqslant \bar{a}_{l}, \quad y \in \Omega_{y}, l \geqslant 0 .
$$

(in this case we say $\bar{a}$ majorizes a). Then

$$
\begin{aligned}
& \left|(a(u(\xi, \eta, y), y))_{m n}\right|_{H_{m+n-1}} \\
& \quad \leqslant \frac{1}{(m+1)^{2}} \frac{1}{(n+1)^{2}}\left(\begin{array}{c}
m+n \\
n
\end{array}\right)\left(\bar{a}\left(K_{0} T_{0} a(z)\right)\right)_{m+n}, \quad m+n \geqslant 1 .
\end{aligned}
$$

Proof. By Corollary 1,

$$
\begin{gathered}
\left|\left(\left(u-u_{00}\right)^{\alpha}\right)_{m n}\right|_{H_{m+n-1}} \leqslant \frac{\left(T_{0} K_{0}\right)^{|\alpha|}}{(m+1)^{2}(n+1)^{2}}\left(\begin{array}{c}
m+n \\
n
\end{array}\right)\left((a(z))^{|\alpha|}\right)_{m+n} \\
\text { since }\left(u-u_{00}\right)_{00}=0 \text {. Hence, } \\
\left(d_{y} \delta\right)^{m+n-1}\left|a(u, y)_{m, n}\right| \leqslant \sum_{l=0}^{\infty} \sum_{|\alpha|=l}\left|a_{\alpha}\left(u_{00}, y\right)\right|\left|\left(u-u_{00}\right)_{m, n}^{\alpha}\right|_{H_{m+n-1}} \\
\leqslant \sum_{l=0}^{\infty} \frac{\left(K_{0} T_{0}\right)^{l}}{(m+1)^{2}(n+1)^{2}}\left(\begin{array}{c}
m+n \\
n
\end{array}\right)\left(a^{l}\right)_{m+n} \sum_{|\alpha|=l}\left|a_{\alpha}\left(u_{00}, y\right)\right| \\
\leqslant \frac{1}{(m+1)^{2}(n+1)^{2}}\left(\begin{array}{c}
m+n \\
n
\end{array}\right)\left(\bar{a}\left(K_{0} T_{0} a\right)\right)_{m+n} .
\end{gathered}
$$

We are now ready to prove the second inequality of the Main Majorization Lemma (see (4.5)).

We consider the terms that enter in $\left(F_{i}\right)_{m n}$ from (2.12). By the remark following (2.12) and by the hypothesis of the Main Majorization Lemma, $u, \phi$ and $\psi$ in these terms satisfy (4.4). In what follows, $m+n=k-1 \geqslant 0$ and $\mu \leqslant m, \nu \leqslant n$.

First, we remark that

$$
u_{\mu \nu}=\sum_{i=1}^{m}\left(\alpha_{i}\right)_{\mu \nu} r_{i}\left(u_{00}\right), \quad u_{\mu}(s, y)=\sum_{i=1}^{m}\left(\alpha_{i}\right)_{\mu} r_{i}\left(u_{0}\right)
$$

so that $\left|u_{\mu \nu}\right| \leqslant T_{0}\left|\alpha_{\mu \nu}\right|,\left|u_{\mu}\right| \leqslant T_{0}\left|\alpha_{\mu}\right|$ with $T_{0}=T_{0}\left(u_{-}, f_{i}\right)$. By (4.4),

$$
\left|\left(\psi_{\eta}-\lambda_{\psi}\right)_{\mu}\right|_{H_{\mu-1}}=\left|(\mu+1) \psi_{\mu+1}\right|_{H_{\mu-1}} \leqslant \frac{1}{(\mu+1)^{2}} a_{\mu} \quad \text { for } 1 \leqslant \mu \leqslant m .
$$

Since $\psi$ does not depend on $\xi,\left(\psi_{\eta}-\lambda_{\psi}\right)_{\mu \nu}=0$ if $\nu>0$. Therefore,

$$
\left|\left(\psi_{\eta}-\lambda_{\psi}\right)\right|_{H_{\mu+\nu-1}} \leqslant \frac{1}{(\mu+1)^{2}} \frac{1}{(\nu+1)^{2}}\left(\begin{array}{c}
\mu+\nu \\
\nu
\end{array}\right) a_{\mu+\nu} \quad \text { for } 1 \leqslant \mu+\nu \leqslant m+n .
$$

Similarly,

$$
\left|\left(\lambda_{\phi}-\phi_{\xi}\right)\right|_{H_{\mu+\nu-1}} \leqslant \frac{1}{(\mu+1)^{2}} \frac{1}{(\nu+1)^{2}}\left(\begin{array}{c}
\mu+\nu \\
\nu
\end{array}\right) a_{\mu+\nu} \quad \text { for } 1 \leqslant \mu+\nu \leqslant m+n
$$


Let $e_{j}$ be the $j$ th unit vector in $R^{n}$. Let $\bar{a}$ be a majorant to

$$
l_{i}\left(u_{00}\right)\left(A(u, y)-A\left(u_{00}, y\right)\right) e_{j} \text { for } i=1, \ldots, m, j=1, \ldots, n,
$$

in the sense of Corollary 2. Then $\bar{a}$ is analytic in some neighborhood of 0 and $\bar{a}(0)=0$.

By Corollary 2,

$$
\begin{aligned}
& \left|\left(l_{i}\left(A(u)-A\left(u_{00}\right)\right) e_{j}\right)_{\mu \nu}\right|_{H_{\mu+\nu-1}} \\
& \quad \leqslant \frac{1}{(\mu+1)^{2}} \frac{1}{(\nu+1)^{2}}\left(\begin{array}{c}
\mu+\nu \\
\nu
\end{array}\right)\left(\bar{a}\left(K_{0} T_{0} a\right)\right)_{\mu+\nu} \quad \text { for } 1 \leqslant \mu+\nu \leqslant m+n .
\end{aligned}
$$

From (4.4),

$$
\left|(\mu+\nu+1)(\psi+\phi)_{\mu \nu}\right|_{H_{\mu+\nu-2}} \leqslant \frac{1}{(\mu+1)^{2}} \frac{1}{(\nu+1)^{2}}\left(\begin{array}{c}
\mu+\nu \\
\nu
\end{array}\right) a_{\mu+\nu-1}
$$

for $2 \leqslant \mu+\nu \leqslant m+n$,

since if both $\mu>0, \nu>0$, the left-hand side is 0 . By Lemma 1 and the above,

$$
\begin{aligned}
& \left|\left((\psi+\phi)_{y_{i}}\right)_{\mu \nu}\right|_{H_{\mu+\nu-1}} \leqslant e(\mu+\nu+1)\left|(\psi+\phi)_{\mu \nu}\right|_{H_{\mu+\nu-2}} \\
& \leqslant \frac{e}{(\mu+1)^{2}(\nu+1)^{2}}\left(\begin{array}{c}
\mu+\nu \\
\nu
\end{array}\right) a_{\mu+\nu-1}=\frac{e}{(\mu+1)^{2}(\nu+1)^{2}}\left(\begin{array}{c}
\mu+\nu \\
\nu
\end{array}\right)(z a)_{\mu+\nu} \\
& \text { for } 2 \leqslant \mu+\nu \leqslant m+n .
\end{aligned}
$$

Since $\left|\left((\psi+\phi)_{y_{i}}\right)_{\mu \nu}\right| \leqslant a_{0}$ if $\mu+\nu=1$ we obtain

$$
\begin{aligned}
\left|\left((\psi+\phi)_{y_{i}}\right)_{\mu \nu}\right|_{H_{\mu+\nu-1}} \leqslant \frac{2 e}{(\mu+1)^{2}(\nu+1)^{2}}\left(\begin{array}{c}
\mu+\nu \\
\nu
\end{array}\right)\left(z a+z a_{0}\right)_{\mu+\nu} & \\
& \text { for } 1 \leqslant \mu+\nu \leqslant m+n .
\end{aligned}
$$

REMARK. If $\mu+\nu=1$, say $\mu=1$ and $\nu=0$, then (4.25) is simply

$$
\left|\left(\psi_{y_{i}}\right)_{1}\right| \leqslant\left(2 e / 2^{2}\right) a_{0}
$$

which holds by definition of $a_{0}$.

Let $\bar{b}$ be a majorant to $l_{i}\left(u_{00}\right) B_{q}=e_{j}$ for all $i, j, q$. By Corollary 2,

$$
\left|\left(l_{i} B_{q} e_{j}\right)_{\mu \nu}\right|_{H_{\mu+\nu-1}} \leqslant \frac{1}{(\mu+1)^{2}(\nu+1)^{2}}\left(\begin{array}{c}
\mu+\nu \\
\nu
\end{array}\right) \bar{b}\left(K_{0} T_{0} a\right)_{\mu+\nu}
$$

$$
\text { for } 1 \leqslant \mu+\nu \leqslant m+n
$$

Also $\left|\left(l_{i} B_{q} e_{j}\right)_{00}\right| \leqslant \bar{b}_{0}$. Next,

$$
\begin{aligned}
&\left|\left(u_{j \xi}\right)_{\mu, \nu}\right|_{H_{\mu+\nu}}=(\mu+1)\left|\left(u_{j}\right)_{\mu+1, \nu}\right|_{H_{\mu+\nu}} \\
& \leqslant T_{0}(\mu+1) \frac{1}{(\mu+2)^{2}} \cdot \frac{1}{(\nu+1)^{2}}\left(\begin{array}{c}
\mu+\nu+1 \\
\nu
\end{array}\right) a_{\mu+\nu+1} \\
& \leqslant \frac{T_{0}}{(\mu+1)^{2}} \frac{1}{(\nu+1)^{2}} \frac{(\mu+\nu+1) !}{\mu ! \nu !}\left(\frac{1}{z} a\right)_{\mu+\nu} \\
& \quad \text { for } 0 \leqslant \mu+\nu \leqslant m+n-1 .
\end{aligned}
$$


Similarly,

$$
\begin{aligned}
\left|\left(u_{j \eta}\right)_{\mu, \nu}\right|_{H_{\mu+\nu}} \leqslant \frac{T_{0}}{(\mu+1)^{2}} \frac{1}{(\nu+1)^{2}} \frac{(\mu+\nu+1) !}{\mu ! \nu !}\left(\frac{1}{z} a\right)_{\mu+\nu} \\
\text { for } 0 \leqslant \mu+\nu \leqslant m+n-1 .
\end{aligned}
$$

Finally,

$$
\begin{aligned}
&\left|\frac{\partial}{\partial y_{q}}\left(u_{j}\right)_{\mu \nu}\right|_{H_{\mu+\nu}} \leqslant e(\mu+\nu+1)\left|\left(u_{j}\right)_{\mu, \nu}\right|_{H_{\mu+\nu-1}} \\
& \leqslant e(\mu+\nu+1) \frac{T_{0}}{(\mu+1)^{2}(\nu+1)^{2}}\left(\begin{array}{c}
\mu+\nu \\
\nu
\end{array}\right) a_{\mu+\nu} \\
&=\frac{e T_{0}}{(\mu+1)^{2}(\nu+1)^{2}} \frac{(\mu+\nu+1) !}{\mu ! \nu !} a_{\mu+\nu} \\
& \text { for } 1 \leqslant \mu+\nu \leqslant m+n,
\end{aligned}
$$

and $\left|\left(\partial / \partial y_{q}\right)\left(u_{j}\right)_{0,0}\right| \leqslant a_{0}$.

We now have

$$
\begin{aligned}
& \left|\left(l_{i}\left\{\left(\psi_{\eta}-\lambda_{\psi}\right)-\left(A(u)-A\left(u_{00}\right)\right)+(\psi+\phi)_{y} B\right\} e_{j}\right)_{\mu \nu}\right|_{H_{\mu+\nu-1}} \\
& \leqslant \frac{1}{(\mu+1)^{2}(\nu+1)^{2}}\left(\begin{array}{c}
\mu+\nu \\
\nu
\end{array}\right) \\
& \quad \times\left\{\sup _{y}\left|l_{i} e_{j}\right| a_{\mu+\nu}+\bar{a}\left(K_{0} T_{0} a\right)_{\mu+\nu}+2 e d \cdot\left(\left(z a+z a_{0}\right) \bar{b}\left(K_{0} T_{0} a\right)\right)_{\mu+\nu}\right\}, \\
& \quad \text { for } 1 \leqslant \mu+\nu \leqslant m+n,
\end{aligned}
$$

where we used (4.23a), (4.24)-(4.26) and (4.19) and summed over $q=1$ to $d$. Also note the left-hand side term is 0 when $\mu=\nu=0$. Hence by (4.20a) applied to (4.27) and the above, summing over $j$, we obtain

(4.29a) $\mid\left(\left.l_{i} \cdot(\text { first term in (2.12)) })_{m n}\right|_{H_{m+n}}\right.$

$$
\begin{aligned}
\leqslant & \frac{C_{0}}{(m+1)^{2}(n+1)^{2}} \frac{(m+n+1) !}{m ! n !} \\
& \cdot\left\{\frac{1}{z} a^{2}+\frac{1}{z} a \cdot \bar{a}\left(K_{0} T_{0} a\right)+\frac{1}{z} \cdot\left(a+a_{0} z\right) \cdot \bar{b}\left(K_{0} T_{0} a\right)\right\}_{m+n} \\
= & \frac{1}{(m+1)^{2}(n+1)^{2}} \frac{(m+n+1) !}{m ! n !}(Q(a)+z R(a, z))_{m+n+1},
\end{aligned}
$$

where

$$
\begin{gathered}
Q(a)=C_{0} a^{2}+a \cdot \bar{a}\left(K_{0} T_{0} a\right), \\
R(a, z)=a \cdot\left(a+a_{0}\right) \cdot \bar{b}\left(K_{0} t_{0} a\right) .
\end{gathered}
$$


Similarly,

(4.29b) $\mid\left. l_{i} \cdot(\text { second term in }(2.12))_{m n}\right|_{H_{m+n}}$

$$
\leqslant \frac{1}{(m+1)^{2}(n+1)^{2}} \frac{(m+n+1) !}{m ! n !}(Q+z R)_{m+n+1} .
$$

It remains to bound the last term in (2.12). From (4.23a) and (4.23b),

$$
\left|\left(\psi_{\eta}-\phi_{\xi}\right)_{\mu \nu}\right|_{H_{\mu+\nu-1}} \leqslant \frac{1}{(\mu+1)^{2}} \frac{1}{(\nu+1)^{2}}\left(\begin{array}{c}
\mu+\nu \\
\nu
\end{array}\right) a_{\mu+\nu}
$$

$$
\text { for } 1 \leqslant \mu+\nu \leqslant m+n,
$$

and $\left|\left(\psi_{\eta}-\phi_{\xi}\right)_{00}\right| \leqslant 2 a_{0}$. Hence by (4.19) and (4.26),

$$
\begin{gathered}
\left|\left(\left(\psi_{\eta}-\phi_{\xi}\right) l_{i} B_{q} e_{j}\right)_{\mu \nu}\right|_{H_{\mu+\nu-1}} \\
\leqslant \frac{K_{0}^{2}}{(\mu+1)^{2}(\nu+1)^{2}}\left(\begin{array}{c}
\mu+\nu \\
\nu
\end{array}\right)\left(\left(a+2 a_{0}\right) \bar{b}\left(K_{0} T_{0} a\right)\right)_{\mu+\nu} \\
\quad \text { for } 1 \leqslant \mu+\nu \leqslant m+n,
\end{gathered}
$$

and $\left|\left(\left(\psi_{\eta}-\phi_{\xi}\right) l_{i} B_{q} e_{j}\right)_{00}\right| \leqslant 2 a_{0} \bar{b}_{0}$.

Applying (4.20) to (4.29) and the above and summing, we obtain

$\mid\left. l_{i} \cdot(\text { last term in }(2.12))_{m n}\right|_{H_{m+n}}$

$$
\begin{aligned}
& \leqslant \frac{C_{0}}{(m+1)^{2}(n+1)^{2}} \frac{(m+n+1) !}{m ! n !}\left(\left(a+a_{0}\right)\left(a+2 a_{0}\right) \bar{b}\left(K_{0} T_{0} a\right)\right)_{m+n} \\
& =\frac{1}{(m+1)^{2}(n+1)^{2}} \frac{(m+n+1) !}{m ! n !}(z R(a(z), z))_{m+n+1}
\end{aligned}
$$

with $R=C_{0}\left(a+a_{0}\right)\left(a+2 a_{0}\right) \bar{b}$. The estimate (4.30) holds for $m+n=0$ as well by the definition of $a_{0}$. Now, (4.29a), (4.29b), and (4.30) together yield the second estimate in (4.5).

REMARKS. When $m=n=0$ the last term in (2.12) is the only nonzero term and (4.30) gives a bound for it.

Also, we have not considered the terms in the end gaps (2.15) separately since they have the same form as (2.12).

LEMMA 8. Let

$$
\begin{gathered}
u(s, t, y)=\sum_{m \geqslant 1} u_{m}(s, y) t^{m}, \text { i.e. } u_{0}=0 \\
v(s, t, y)=\sum_{m \geqslant 0} v_{m}(s, y) t^{m}, \quad w(s, t, y)=\sum_{m \geqslant 0} w_{m}(s, y) t^{m} .
\end{gathered}
$$

Consider the following estimates:

$$
\left|u_{m}\right|_{H_{m-1}} \leqslant\left(T_{0} /(m+1)^{2}\right) a_{m}, \quad m \geqslant 1,
$$




$$
\begin{gathered}
\left|w_{m}\right|_{H_{m-1}} \leqslant\left(T_{1} /(m+1)^{2}\right) b_{m}, \quad m \geqslant 1, \\
\left|v_{m}\right|_{H_{m}} \leqslant\left(T_{2}(m+1) /(m+1)^{2}\right) c_{m}, \quad m \geqslant 0,
\end{gathered}
$$

with $a_{m}, b_{m}, c_{m} \geqslant 0,\left|w_{0}\right|_{H_{0}} \leqslant T_{1} b_{0}$. Then

(A) If (i) holds for $1 \leqslant m \leqslant k$ and (ii) holds for $1 \leqslant m \leqslant k-1$ then

$$
\left|(u w)_{k}\right|_{H_{k-1}} \leqslant\left(T_{0} T_{2} K_{0}^{2} /(k+1)^{2}\right)\left[(a(z))\left(\mathscr{b}(z)+b_{0}\right)\right]_{k} \text {. }
$$

If (i) holds for $1 \leqslant m \leqslant k-1$, then

$$
\left|\left(u^{2}\right)_{k}\right|_{H_{k-1}} \leqslant\left(T_{0}^{2} K_{0}^{2} /(k+1)^{2}\right)\left(a^{2}(z)\right)_{k} .
$$

(B) If Case 1: (i) holds for $1 \leqslant m \leqslant k$ and (iii) holds for $0 \leqslant m \leqslant k-1$; or Case 2: (i) holds for $1 \leqslant m \leqslant k-1$ and $v_{0}=0=c_{0}$ and (iii) holds for $1 \leqslant m \leqslant k-1$ then

$$
\left|(u v)_{k}\right|_{H_{k-1}} \leqslant\left(T_{0} T_{1} K_{0}^{2} /(k+1)^{2}\right)(k+1)(a \cdot c)_{k} .
$$

(C) If $u_{1}=0=a_{1}$ and (i) holds for $2 \leqslant m \leqslant k$ and (iii) holds for $0 \leqslant m \leqslant k-2$, then

$$
\left|(u v)_{k}\right|_{H_{k-1}} \leqslant\left(T_{0} T_{1} K_{0}^{2} /(k+1)^{2}\right)(k+1)[a(z) \cdot c(z)]_{k} .
$$

Proof. The results in (A) and (B) follow from Lemma 7(4.19a), (4.20a), respectively, by considering only one index, say $\mu \leqslant m, m=k, \nu=n=0$. Part (C) is almost immediate:

$$
\begin{aligned}
\left|(u v)_{k}\right|_{H_{k-1}} & \leqslant \sum_{m=2}^{k}\left|u_{m}\right|_{m-1}\left|v_{k-m}\right|_{k-m} \leqslant \frac{T_{0} T_{1} K_{0}^{2}(k+1)}{(k+1)^{2}} \sum_{m=2}^{k} a_{m} c_{k-m} \\
& =\frac{T_{0} T_{1} K_{0}^{2}(k+1)}{(k+1)^{2}}(a \cdot c)_{k} \quad \text { if } a_{1}=0 .
\end{aligned}
$$

COROllary 3. Let $u=\left(u_{1}, \ldots, u_{n}\right)$ with $u_{i}(s, t, y)=\sum_{m \geqslant 0} u_{i_{m}}(s, y) t^{m}$ and $u_{i_{m}}$ satisfy (i) of Lemma 8 for $1 \leqslant m \leqslant \mu$. Let $a(u, y)=\sum_{\alpha} a_{\alpha}\left(u_{0}, y\right)\left(u-u_{0}\right)^{\alpha}$ be an analytic function in the variables $(u, y)$ and suppose

$$
\sum_{|\alpha|=l}\left|a_{\alpha}\left(u_{0}(s, y), y\right)\right| \leqslant \bar{a}_{l}, \quad(s, y) \in \Omega_{s} \times \Omega_{y},
$$

where $u_{0}(s, y)=u(s, 0, y)$. Then

$$
\left|(a(u(s, t, y), y))_{\mu}\right|_{H_{k-1}} \leqslant(\mu+1)^{-2}\left(\bar{a}\left(K_{0}^{2} T_{0} a(z)\right)\right)_{\mu} .
$$

Proof.

$$
\left|(a(u, y))_{\mu}\right|_{H_{\mu-1}} \leqslant \frac{1}{(\mu+1)^{2}} \sum_{l \geqslant 0} \bar{a}_{l} T_{0}^{\prime}\left(K_{0}^{2}\right)^{l}\left(a^{\prime}(z)\right)_{\mu} .
$$

Corollary 3 follows.

REMARKs. If $|\alpha| \geqslant 2$ it follows by (4.31a) that

$$
\left|\left(\left(u-u_{0}\right)^{\alpha}\right)_{\mu}\right|_{H_{\mu-1}} \leqslant \frac{T_{0}^{l}\left(K_{0}^{2}\right)^{l-1}}{(\mu+1)^{2}}\left(a^{\prime}(z)\right)_{\mu}
$$


under the weaker hypothesis that $\left(u_{i}\right)_{m}$ satisfies (i) for $1 \leqslant m \leqslant \mu-1$. Therefore, if $a_{\alpha}=0$ for $|\alpha|<2$, Corollary 3 is valid under this weaker hypothesis.

We now focus our attention on (2.17), the inhomogeneous term in rarefactions. We let $a(z)$ be as in the hypothesis of the Main Majorization Lemma.

We have from (4.4)

$$
\begin{gathered}
\left|\left(\alpha_{i_{t}}\right)_{\mu}\right|_{H_{\mu}}=(\mu+1)\left|\left(\alpha_{i}\right)_{\mu+1}\right|_{H_{\mu}} \leqslant(\mu+1) \frac{1}{(\mu+2)^{2}} a_{\mu+1} \\
\leqslant \frac{\mu+1}{(\mu+1)^{2}}\left(\frac{1}{z} a(z)\right)_{\mu} \text { for } 0 \leqslant \mu \leqslant k-2 . \\
\left|\left(\psi-\lambda_{\psi} t\right)_{\mu}\right|_{H_{\mu-1}} \leqslant\left|\left(\psi-\lambda_{\psi} t\right)_{\mu}\right|_{H_{\mu-2}} \\
\leqslant \frac{1}{\mu} \frac{1}{\mu^{2}} a_{\mu-1} \leqslant \frac{4}{(\mu+1)^{2}}(z a)_{\mu} \text { for } 2 \leqslant \mu \leqslant k,
\end{gathered}
$$

and $\left(\psi-\lambda_{\psi} t\right)_{0}=\left(\psi-\lambda_{\psi} t\right)_{1}=0$.Similarly,

$$
\left|\left(\phi-\psi_{\phi} t\right)_{\mu}\right|_{H_{\mu-1}} \leqslant \frac{4}{(\mu+1)^{2}}(z a)_{\mu} \text { for } 2 \leqslant \mu \leqslant k .
$$

Using (4.31c) on (4.32)-(4.34) we obtain

$$
\left|\left(\left(\left(\psi-\lambda_{\psi} t\right)-\left(\phi-\lambda_{\phi} t\right)\right) \alpha_{i_{t}}\right)_{k}\right|_{H_{k-1}} \leqslant \frac{C_{0}(k+1)}{(k+1)^{2}}\left(a^{2}\right)_{k} .
$$

From Corollary 3,

$$
\left|\left(l_{i} \cdot\left(A(u)-A\left(u_{0}\right)\right) e_{j}\right)_{\mu}\right|_{H_{\mu-1}} \leqslant \frac{1}{(\mu+1)^{2}} \bar{a}\left(C_{0} a\right)_{\mu} \quad \text { for } 1 \leqslant \mu \leqslant k-1
$$

with $\bar{a}$ a majorant for $l_{i}\left(A-A\left(u_{0}\right)\right) e_{j}$ and $C_{0}=K_{0}^{2} T_{0}$ in this case. Also,

$$
\left|\left(\left(u_{j}-u_{j_{0}}\right)_{s}\right)_{\mu}\right|_{H_{\mu}} \leqslant e(\mu+1)\left|\left(u_{j}-u_{j_{0}}\right)_{\mu}\right|_{H_{\mu-1}} \leqslant C_{0} \frac{(\mu+1)}{(\mu+1)^{2}} a_{\mu}
$$

for $1 \leqslant \mu \leqslant k-1$.

Using (4.31b) Case 2 in Lemma 8 applied to the above two estimates and summing over $j$, we obtain

$$
\left|\left(l_{i}\left(A(u)-A\left(u_{0}\right)\right)\left(u-u_{0}\right)_{s}\right)_{k}\right|_{H_{k-1}} \leqslant \frac{C_{0}(k+1)}{(k+1)^{2}}\left(\bar{a}\left(C_{0} a\right) a\right)_{k} .
$$

Note that $\bar{a}(0)$ may be taken to be zero.

Since

$$
A(u)-A\left(u_{0}\right)-A^{\prime}\left(u_{0}\right)\left(u-u_{0}\right)=\sum_{|\alpha| \geqslant 2} A_{\alpha}\left(u_{0}\right)\left(u-u_{0}\right)^{\alpha},
$$

if we let $\bar{a}_{Q}=\sum_{j \geqslant 2} \bar{a}_{j} z^{j}$, we then have that $\bar{a}_{Q}$ is a majorant for

$$
l_{i}\left(A(u)-A\left(u_{0}\right)-A^{\prime}\left(u_{0}\right)\left(u-u_{0}\right)\right) e_{j} .
$$


Hence, Corollary 3 and the remark following it imply

$$
\left|\left(l_{i}\left(A-A\left(u_{0}\right)-A^{\prime} \cdot\left(u-u_{0}\right)\right) u_{0_{s}}\right)_{k}\right|_{H_{k-1}} \leqslant \frac{C_{0}}{(k+1)^{2}} \bar{a}_{Q}\left(C_{0} a\right)_{k},
$$

where $C_{0}$ here is also a bound for $\left|u_{0_{s}}\right|$.

Since

$$
\left|\left(\phi_{t}-\lambda_{\phi}\right)\right|_{H_{\mu-1}}=\left|(\mu+1) \phi_{\mu+1}\right|_{H_{\mu-1}} \leqslant \frac{1}{(\mu+1)^{2}} a_{\mu} \quad \text { for } 1 \leqslant \mu \leqslant k-1
$$

and since

$$
\left|\left(l_{i}\left(u-u_{0}\right)_{s}\right)_{\mu}\right|_{H_{\mu}} \leqslant \frac{C_{0}(\mu+1)}{(\mu+1)^{2}} a_{\mu} \text { for } 1 \leqslant \mu \leqslant k-1,
$$

by Case 2 of (B) in Lemma 8 we obtain

$$
\begin{gathered}
\left|\left[\left(\phi_{t}-\lambda_{\phi}+s\left(\left(\psi_{t}-\lambda_{\psi}\right)-\left(\phi_{t}-\lambda_{\phi}\right)\right)\right) l_{i}\left(u-u_{0}\right)_{s}\right]_{k}\right|_{H_{k-1}} \\
\leqslant \frac{C_{0}(k+1)}{(k+1)^{2}}\left(a^{2}\right)_{k} .
\end{gathered}
$$

Corollary 3 implies

$$
\left|\left(l_{i} B_{q} e_{j}\right)_{\mu}\right|_{H_{\mu-1}} \leqslant(\mu+1)^{-2}\left(\bar{b}\left(C_{0} a\right)\right)_{\mu} \text { for } 0 \leqslant \mu \leqslant k-1
$$

with $\bar{b}$ a majorant for $l_{i} B_{q} e_{j}$. Also,

$$
\left|(\psi-\phi)_{\mu}\right|_{H_{\mu-1}} \leqslant\left|(\psi-\phi)_{\mu}\right|_{H_{\mu-2}} \leqslant \frac{2}{\mu^{3}} a_{\mu-1} \leqslant \frac{4}{(\mu+1)^{2}}(z a)_{\mu}
$$

for $2 \leqslant \mu \leqslant k$.

Since $\left|(\psi-\phi)_{1}\right| \leqslant 2 a_{0}$, we obtain

(4.38c) $\quad\left|(\psi-\phi)_{\mu}\right|_{H_{\mu-1}} \leqslant \frac{4}{(\mu+1)^{2}}\left[\left(z a+2 a_{0} z\right)\right]_{\mu} \quad$ for $1 \leqslant \mu \leqslant k$ and $(\psi-\phi)_{0}=0$. Also, as in (4.29),

$$
\left|\frac{\partial}{\partial y_{q}}\left(u_{j}\right)_{\mu}\right|_{H_{\mu}} \leqslant \frac{e T_{0}}{(\mu+1)^{2}}(\mu+1) a_{\mu} \quad \text { for } 1 \leqslant \mu \leqslant k-1,
$$

with $\left|\left(\partial / \partial y_{q}\right)\left(u_{j}(s, y)\right)_{0}\right| \leqslant a_{0}$. Applying Case 1 of (B) in Lemma 8 to (4.38d) and (4.38c) we get

$$
\left|\left((\psi-\phi) \frac{\partial}{\partial y_{q}} u_{j}\right)_{\mu}\right|_{H_{\mu-1}} \leqslant \frac{C_{0}(\mu+1)}{(\mu+1)^{2}}\left[\left(a+a_{0}\right)\left(z a+2 a_{0} z\right)\right]_{\mu}
$$

$$
\text { for } 1 \leqslant \mu \leqslant k \text {. }
$$

Applying Case 1 of (B) in Lemma 8 one more time to (4.38a) and the above, and summing over $q$ and $j$, we obtain

$$
\left|\left(l_{i} B(\psi-\phi) u_{y}\right)_{k}\right|_{H_{k-1}} \leqslant \frac{C_{0}(k+1)}{(k+1)^{2}}\left[\bar{b}\left(C_{0} a\right) \cdot\left(a+a_{0}\right)\left(z a+2 a_{0} z\right)\right]_{k} .
$$


Since using Lemma 1 and then (4.4),

$$
\begin{aligned}
\left|\left(\phi_{y_{q}}+s(\psi-\phi)_{y_{q}}\right)_{\mu}\right|_{H_{\mu-1}} & \leqslant e(\mu-1)\left|\phi+s(\psi-\phi)_{\mu}\right|_{H_{\mu-2}} \\
\leqslant & \frac{C_{0}(\mu-1)}{\mu} \cdot \frac{1}{\mu^{2}} a_{\mu-1} \leqslant \frac{C_{0}}{(\mu+1)^{2}}(z a)_{\mu} \quad \text { for } 2 \leqslant \mu \leqslant k,
\end{aligned}
$$

and since $\left|\left(\phi_{y_{q}}+s(\psi-\phi)_{y_{q}}\right)_{1}\right| \leqslant C_{0} a_{0}$, we obtain

(4.40) $\left|\left(\phi_{y_{q}}+s(\psi-\phi)_{v_{q}}\right)_{\mu}\right|_{H_{\mu-1}} \leqslant C_{0}(\mu+1)^{-2}\left(z a+a_{0} z\right)_{\mu} \quad$ for $1 \leqslant \mu \leqslant k$.

Also,

$$
\left|\frac{\partial}{\partial s}\left(u_{j}\right)_{\mu}\right|_{H_{\mu}} \leqslant \frac{T_{0} e(\mu+1)}{(\mu+1)^{2}}\left(a+a_{0}\right)_{\mu} \text { for } 0 \leqslant \mu \leqslant k-1 .
$$

Applying Lemma 8, part (B), Case 1 to (4.40) and the above, we obtain

$$
\left|\left[\left(\phi_{y_{q}}+s(\psi-\phi)_{y_{q}}\right)\left(u_{j}\right)_{s}\right]_{\mu}\right|_{H_{\mu-1}} \leqslant \frac{C_{0}(\mu+1)}{(\mu+1)^{2}}\left[\left(a+a_{0}\right)\left(z a+a_{0} z\right)\right]_{\mu}
$$

for $1 \leqslant \mu \leqslant k$.

Applying Lemma 8 one more time to (4.38a) and the above, and summing over $q$ and $j$, we obtain

$$
\begin{aligned}
& \left|\left(l_{i} \cdot B \cdot\left(\phi_{y}+s(\psi-\phi)_{y}\right) u_{s}\right)_{k}\right|_{H_{k-1}} \\
& \quad \leqslant \frac{C_{0}(k+1)}{(k+1)^{2}}\left[\left(a+a_{0}\right) \cdot\left(z a+a_{0} z\right) \cdot \bar{b}\left(C_{0} a\right)\right]_{k} .
\end{aligned}
$$

Collecting the results from (4.35)-(4.39) (4.41), we obtain the first estimate in (4.5).

We consider (2.22) next. From (4.4),

$$
\begin{gathered}
\left|\left(\phi_{t}-\lambda_{\phi}\right)_{\mu}\right|_{H_{\mu-1}} \leqslant(\mu+1)^{-2} a_{\mu} \text { for } 1 \leqslant \mu \leqslant k-1 \\
\left|\left(\left(u-u_{00}\right)-\left(v-v_{00}\right)\right)_{\mu}\right|_{H_{\mu-1}} \leqslant 2 T_{0}(\mu+1)^{-2} a_{\mu} \quad \text { for } 1 \leqslant \mu \leqslant k-1 .
\end{gathered}
$$

Hence Lemma 8 (4.31a) implies

$$
\left|l_{i}\left[\left(\phi_{t}-\lambda_{\phi}\right)\left(\left(u-u_{00}\right)-\left(v-v_{00}\right)\right)\right]_{k}\right|_{H_{k-1}} \leqslant \frac{C_{0}}{(k+1)^{2}}\left(a^{2}\right)_{k} .
$$

Next, using Corollary 3, we obtain

$$
\begin{aligned}
\mid l_{i}\left[\left(f_{0}(u, y)-f_{0}\left(u_{00}, y\right)-A\left(u_{00}\right)\left(u-u_{00}\right)\right)\right. & \\
& \left.-\left(f_{0}(v, y)-f_{0}\left(v_{00}, y\right)-A\left(v_{00}\right)\left(v-v_{00}\right)\right)\right]\left.\right|_{H_{k-1}} \\
\leqslant & \frac{1}{(k+1)^{2}}\left(\bar{f}_{0 Q}\left(C_{0} a(z)\right)\right)_{k}
\end{aligned}
$$

where $\bar{f}_{0 Q}=\sum_{l \geqslant 2} \bar{f}_{0 l} z^{l}$ with $\bar{f}_{0}$ a majorant for $l_{i} \cdot f_{0}$ at $u_{00}$ and $v_{00}$.

REMARKS. Since $f_{0}^{\prime}=A$ the left-hand side of (4.43) is quadratic in $\left(u-u_{00}\right)$. 
Using Corollary 3 we also get

$$
\left|\left(l_{i} \cdot\left(f_{q}(u)-f_{q}(v)\right)\right)_{\mu}\right|_{H_{\mu-1}} \leqslant\left((\mu+1)^{-2}\right)\left(\bar{f}\left(C_{0} a(z)\right)\right)_{\mu} \quad \text { for } 0 \leqslant \mu \leqslant k-1
$$

where $\bar{f}$ is a majorant for $l_{i} \cdot f_{q}, q=1, \ldots, d$. Since

(4.43a) $\left|\left(\phi_{y}\right)_{\mu}\right|_{H_{\mu-1}} \leqslant \frac{C_{0}}{(\mu+1)^{2}}\left(z a+a_{0} z\right)_{\mu} \quad$ for $1 \leqslant \mu \leqslant k \quad$ (see (4.40))

by Lemma $8(4.31)$

$$
\text { (4.44) }\left|\left(l_{i}(f(u)-f(v)) \cdot \phi_{y}\right)_{k}\right|_{H_{k-1}} \leqslant C_{0}(k+1)^{-2}\left(\bar{f}\left(C_{0} a(z)\right) \cdot\left(z a+a_{0} z\right)\right)_{k} \text {. }
$$

Putting (4.42)-(4.44) together we obtain the third estimate in (4.5).

The expressions for $L_{j}$, and $L_{p}$ from (2.25a) and (2.29), respectively, are similar so it suffices to restrict our attention to $L_{p}$ in (2.29). Let

$$
\lambda_{p}\left(u, y, \phi_{y}\right)=\sum_{\alpha, j}\left(\lambda_{p}\right)_{\alpha, j}\left(u_{00}, y, 0\right)\left(u-u_{00}\right)^{\alpha} \phi_{y}^{j} .
$$

Then

(4.45) $L_{p}\left(u, y, \phi_{y}\right)=\sum_{\substack{|\alpha| \geqslant 2 \\ j=0}}\left(\lambda_{p}\right)_{\alpha, 0}\left(u-u_{00}\right)^{\alpha}+\sum_{\substack{\alpha \\ j \geqslant 1}}\left(\lambda_{p}\right)_{\alpha, j} \cdot\left(u-u_{00}\right)^{\alpha} \phi_{y}^{j}$.

Let $\bar{\lambda}_{p}(z, w)$ be a majorant of $\lambda_{p}$, that is $\sum_{|\alpha|=l}\left|\left(\lambda_{p}\right)_{\alpha, j}\left(u_{00}(y), y\right)\right| \leqslant\left(\bar{\lambda}_{p}\right)_{l, j}$, $y \in \Omega_{y}$. Since

$$
\left|\left(\phi_{y}^{j}\right)_{\mu}\right|_{H_{\mu-1}} \leqslant C_{0}^{j}(\mu+1)^{-2}\left(\left(z a+a_{0} z\right)^{j}\right)_{\mu} \text { for } 1 \leqslant \mu \leqslant k,
$$

(see (4.43a) and Lemma 8 (4.31a)) and by Lemma 8 (4.31a)

$$
\left|\left(\left(u-u_{00}\right)^{\alpha}\right)_{\mu}\right|_{H_{\mu-1}} \leqslant C_{0}^{|\alpha|}(\mu+1)^{-2}\left(a^{|\alpha|}\right)_{\mu} \text { for } 0 \leqslant \mu \leqslant k-1,|\alpha| \geqslant 0,
$$

we obtain by Lemma 8 (4.31)

$$
\left|\left(\left(u-u_{00}\right)^{\alpha} \phi_{y}^{j}\right)_{k}\right|_{H_{k-1}} \leqslant \frac{C_{0}^{j} C_{0}^{|\alpha|}}{(k+1)^{2}}\left(a^{|\alpha|}\left(z a+a_{0} z\right)^{j}\right)_{k} .
$$

Hence we may estimate the terms in (4.45) by

$$
\begin{aligned}
\mid\left(\sum_{j \geqslant 1}\left(\lambda_{p}\right)_{\alpha, j}\left(u-u_{00}\right)^{\alpha} \phi_{y}^{j}\right) & \left.\left.\right|_{k}\right|_{H_{k-1}} \\
& \leqslant \frac{1}{(k+1)^{2}} \sum_{\substack{j \geqslant 1 \\
l \geqslant 0}}\left(\bar{\lambda}_{p}\right)_{l, j} C_{0}^{j} C_{0}^{l}\left(a^{l}\left(z a+a_{0} z\right)^{j}\right)_{k} \\
& =\frac{1}{(k+1)^{2}} z C_{0}\left[\sum_{\substack{j \geqslant 0 \\
l \geqslant 0}}\left(\bar{\lambda}_{p}\right)_{l, j+1} C_{0}^{j} C_{0}^{l}\left(a^{l}\left(z a+a_{0} z\right)^{j}\right)\right]_{k} \\
& =(k+1)^{2}(z R(a, z))_{k} .
\end{aligned}
$$


Finally, we estimate the first term in (4.45):

$$
\left|\left(\sum_{|\alpha| \geqslant 2}\left(\lambda_{p}\right)_{\alpha, 0} \cdot\left(u-u_{00}\right)^{\alpha}\right)\right|_{k} \leqslant \frac{1}{(k+1)^{2}}\left(\bar{\lambda}_{p Q}\left(C_{0} a\right)\right)_{k} .
$$

The fourth and last estimate in (4.5) follows.

ACKNOWLEDGEMENTS. I would like to thank James Ralston and Stanley Osher for their kind help and encouragement and Georgia Ballance for her expert typing of the manuscript.

\section{REFERENCES}

1. P. D. Lax, Hyperbolic systerms of conservation laws and the mathematical theory of shock waves, SIAM Regional Conf. Ser. in Appl. Math., No. 11, 1973.

2. R. Courant and K. O. Friedrichs, Supersonic flow and shock waves, Springer-Verlag, New York, 1976, pp. 6-8.

3. L. Hörmander, Linear partial differential operators, Springer-Verlag, Berlin, 1963.

4. M. Shinbrot and R. R. Welland, The Cauchy-Kowalewskaya theorem, J. Math. Anal. Appl. 55 (1976), $757-772$.

5. A. Majda, The stability of multi-dimensional shock fronts and The existence of multi-dimensional shock fronts, Mem. Amer. Math. Soc. Nos. 275 and 281, 1983.

6. R. D. Richtmyer, Principles of advanced mathematical physics, Vol. 1, Springer-Verlag, New York, 1978, pp. 393-394.

Institute for Computer Applications in Science and Engineering, Mail Stop 132C, NASA Langley Research Center, Hampton, Virginia 23665

Current address: Department of Mathematics, University of California, Los Angeles, California 90024 